\title{
Institutionnalisme(s), rationalisme et structuralisme en science sociale
}

\section{Bernard Billaudot}

\section{(2) OpenEdition}

\section{Journals}

Édition électronique

URL : http://journals.openedition.org/ei/791

DOI : 10.4000/ei.791

ISSN : 2553-1891

\section{Éditeur}

Association Économie et Institutions

\section{Édition imprimée}

Date de publication : 1 juin 2004

Pagination : 5-50

ISSN : 1775-2329

\section{Référence électronique}

Bernard Billaudot, «Institutionnalisme(s), rationalisme et structuralisme en science sociale »,

Économie et institutions [En ligne], 4 | 2004, mis en ligne le 31 janvier 2013, consulté le 10 décembre 2020. URL : http://journals.openedition.org/ei/791 ; DOI : https://doi.org/10.4000/ei.791

Revue Économie et institutions 


\title{
Institutionnalisme(s), rationalisme et structuralisme en science sociale ${ }^{1}$
}

\author{
Bernard Billaudot 2
}

\section{Introduction}

A partir du début des années mille neuf cent soixante dix, beaucoup d'économistes ont progressivement franchit le Rubicon qui séparait la science économique de la sociologie. Reconnaissant l'existence empirique de règles (normes sociales, réglementations publiques, arrangements organisationnels ou contractuels particuliers), ils se préoccupent de les expliquer ou de les comprendre théoriquement ${ }^{3}$. De même, à l'autre bout du champ académique, pour ceux qui ne se sont plus contenté d'une approche marxiste ne traitant qu'en creux des institutions ${ }^{4}$. En ce sens, les uns et les autres sont devenus des institutionnalistes, sans que tous s'affichent pour autant comme tels et a fortiori revendiquent une filiation avec l'école institutionnaliste américaine du début du XXième siècle.

Ce renouveau de l'institutionnalisme au sens large n'est pas propre à l'économie. On le constate dans toutes les sciences sociales. Des chercheurs se sont préoccupés de classer ces travaux. Sur la base d'un recensement relativement complet de ces derniers en science politique et en théorie des organisations, Hall et Taylor [1996] distinguent trois façons principales de mettre en œuvre une analyse institutionnaliste ou encore trois néoinstitutionnalismes : l'institutionnalisme historique $(\mathrm{IH})$, l'institutionnalisme des choix rationnels, que je propose de qualifier plus simplement d'institutionnalisme rationnel (IR), et l'institutionnalisme sociologique (ISo) ${ }^{5}$.

1 Je dois aux rapporteurs, ainsi qu'aux animateurs de la revue, de m'avoir incité à abandonner comme entrée dans le sujet la petite porte "scientifique " de la méthodologie que j'avais retenue dans une version antérieure pour la grande porte "philosophique " de la vision de la vie des hommes en société. Je les en remercie.

2 LEPII (ex IREPD),CNRS-UPMF Grenoble, bernard.billaudot@upmfgrenoble.fr.

3 Voir notamment [Arrow, 1973, 1974], [North, 1990], [Mäki, Gustafsson, Knudsen, 1993] et [Postel, 2003].

4 En particulier, l'école de la régulation en France [Boyer, Saillard, 2002], [Boyer, 2004].

5 Je le note "ISo ", avec "So " pour " sociologique ", dans la mesure où j'utilise dans la suite "S " pour " structuraliste ". Précisons tout de suite que cette dénomination choisie par Hall et Taylor n'a en rien pour objet de désigner la façon dont les sociologues traitent des institutions. D'ailleurs, à s'en tenir à l'état actuel des programmes de recherche au sein de cette

5 Economie et Institutions $-\mathrm{n}^{\circ} 4-1^{\mathrm{e}}$ semestre 2004 
montrant que cette distinction se retrouve dans toutes les sciences sociales, notamment en économie. Et il l'a systématisée en proposant, en amont de la distinction entre disciplines, une vision du champ des institutionnalismes ce champ est construit en considérant les deux questions retenues par Hall et Taylor pour interroger les écoles existantes et les différencier : (1) comment construire la relation entre institution et comportement, (2) comment expliquer le processus par lequel les institutions naissent et se modifient ${ }^{6}$.

Cet apport relève de la démarche classique consistant à partir des pratiques de recherche et à construire une typologie de ces pratiques. Il s'agit d'une étape incontournable. Mais on en connaît aussi la principale limite: le fond commun de ces trois approches n'est alors défini qu'en extension. Autrement dit, si on se focalise sur les institutionnalismes actuels, ce sont les différences qui ressortent; leur fond commun s'efface derrière celles-ci ou se réduit comme une peau de chagrin [DiMaggio et Powell, 1991], le seul point commun étant de prendre en compte les institutions et de se préoccuper de les ressaisir dans l'analyse [Favereau, 1995]. On doit

discipline, les trois institutionnalismes y sont représentés, notamment l'IR avec J. S. Coleman [1990].

6A chacune de ces questions est associé un axe. Le premier axe oppose le calcul (stratégie) et la culture (routine) comme raisons pour un individu d'adopter un comportement conforme à des règles et le second, la coordination (cognition) et le conflit (pouvoir) comme sources des institutions. Les trois (néo)institutionnalismes ne sont pas les quatre pôles de ce champ à deux axes. Ce sont des positions dans ce champ. La RAT (IR) est proche du premier pôle (calcul-coordination), l'IS (ISo) est proche du second (culture-coordination) et l'IH est en position médiane relativement au premier axe (il combine calcul et culture) tout en se situant du côté du conflit sur le second, ce qui l'oppose à la fois à la RAT et l'IS. S'agissant de positions correspondant à des regroupements de recherches effectives, et non de normes, ces positions sont à même d'évoluer.

$7 \mathrm{La}$ définition que ce dernier donne alors d'une institution comme étant "tout système de règles doté d'une certaine cohérence "[Favereau, 1995 : 511] est celle qui convient. En effet, il s'agit d'une simple désignation empirique, dans laquelle une institution particulière relève d'une délimitation ad hoc en l'absence de critère théorique pour juger de la dite cohérence. Elle est telle que la liste des institutions se décline presque à l'infini, comme un poème de Prévert. Toute tentative de préciser cette définition nous conduit sur le terrain de l'un des institutionnalismes. Tel est notamment le cas de celle de Norh [1990 : 3], pour qui "Les institutions sont les règles $\mathrm{du}$ jeu, dans une société, ou, plus formellement, sont les contraintes d'origine humaine qui encadrent l'interaction humaine. En conséquence elles structurent les incitations dans l'échange humain, qu'il soit politique, social ou économique". En effet, 1/ cette définition présuppose qu'on sait ce qu'est une "société "; 2/ elle affirme que les institutions seraient "contraignantes" et qu'elles "structureraient les

Economie et Institutions $-n^{\circ} 4-1^{e}$ semestre 2004 
recourir à une démarche en compréhension si on entend lever quelque peu le flou et le vague d'une telle définition de ce fond commun et se prononcer en faveur de la thèse selon laquelle il s'agit bien d'une nouvelle problématique générale dite institutionnaliste qui s'affirme. C'est à cette condition que l'on peut comprendre à la fois en quoi cette dernière se distingue des deux problématiques traditionnelles de la science sociale, la problématique rationaliste et la problématique structuraliste, et pourquoi cette nouvelle problématique générale se décline selon trois modalités distinctes et seulement trois 8 .

Cet article développe une telle démarche en compréhension de $l^{\prime}$ institutionnalisme et de sa déclinaison, en se situant à l'amont de toute division du travail entre sciences sociales. Il ne traite pas de l'application de cette grille d'analyse à l'économie. Il comprend trois parties. On commence par présenter l'hypothèse d'une problématique institutionnaliste ayant son identité propre au regard des deux grandes problématiques traditionnelles, le rationalisme et le structuralisme (I). On fait ensuite état des raisons méthodologiques pour lesquelles elle se diffracte en trois approches tout à fait distinctes les unes des autres, en précisant alors les trois visions de la vie des hommes en société qui en sont constitutives (II). On montre enfin que cette déclinaison de la problématique institutionnaliste correspond à trois étapes dans l'appréhension de la complexité de la vie sociale (III). On en conclut que ces trois approches sont à la fois complémentaires et contradictoires.

incitations ", ce qui est déjà un parti pris théorique particulier dans la façon de concevoir une institution; $3 /$ elle laisse entendre que "l'interaction humaine " et "l'échange humain " seraient somme toute la même chose ; on est alors conduit à dire que les institutions seraient associées à la réalisation de la coordination des actions humaines en tant que ces actions visent un échange ; on laisse ainsi dans l'ombre toutes les interactions non voulues, notamment nombre de règles techniques issues de processus de polarisation mimétique.

8 Ceci est d'autant plus nécessaire qu'une partie du flou dont on vient de faire état tient au fait que les deux questions qui servent à organiser l'analyse des pratiques de recherche dans le champ considéré ne sont pas indépendantes. En effet, l'explication que l'on peut donner du processus par lequel les institutions naissent et se modifient dépend essentiellement de la façon dont on construit la relation entre institutions et comportement individuel, de telle sorte que la réponse à la seconde est contenue dans celle que l'on apporte à la première.

7 Economie et Institutions $-\mathrm{n}^{\circ} 4-1^{\mathrm{e}}$ semestre 2004 


\section{L'hypothèse d'une problématique institutionnaliste}

L'objet de la science sociale est la vie des hommes en société ; les phénomènes sociaux que l'on se propose d'expliquer ou de comprendre au point d'aboutissement de la démarche de construction de toute théorie sont des évènements qui sont observés au niveau méso ou macro social ; les actions individuelles, les comportements individuels en société, en sont naturellement exclus; mais aussi les normes, les règles, les arrangements organisationnels particuliers de toute sorte, en un mot les institutions. Les deux grandes problématiques traditionnelles de traitement de cet objet ont en commun de retenir que tout phénomène social est le résultat de comportements individuels en société ; mais elles divergent quant à la façon de voir ces comportements et elles préconisent en conséquence deux principes méthodologiques différents, d'un côté l'individualisme méthodologique et de l'autre le holisme méthodologique. L'hypothèse selon laquelle une nouvelle problématique s'affirme, avec une identité propre, se heurte à une difficulté, apparemment insurmontable : il n'y a pas place pour un troisième principe méthodologique. Le bien fondé de cette hypothèse ne peut donc être établi qu'en se focalisant sur le débat relatif à la vision que l'on peut/doit retenir, c'est à dire sur un réexamen des réponses possibles aux deux questions suivantes. Que voit-on à l'amont des comportements des individus? Comment voit-on la formation de ces objets collectifs, que sont les institutions, à partir de ces comportements lorsqu' on s'en préoccupe ? La première conduit à revenir sur la rationalité d'un comportement et la seconde, sur la vision holiste selon laquelle les ensembles sociétaux (les phénomènes sociaux et les objets collectifs) ne se réduisent pas à la somme des actions individuelles.

Après avoir précisé en quels sens on parle de problématique générale et d'approche de la science sociale, on revient d'abord sur les deux versants du comportement humain. On montre ensuite que l'on ne peut enfermer la rationalité d'un comportement dans sa représentation « rationaliste» et que la vision holiste n'est pas nécessairement «structuraliste ». Cela fournit les bases d'une première identification en compréhension d'une problématique institutionnaliste.

\subsection{Les notions de problématique et d'approche de la science sociale}

Pour Joseph Schumpeter, toute théorie positive - entendue comme une analyse ordonnée de phénomènes observés dans un domaine - repose sur une vision de ce domaine. Ainsi, une vision n'est pas encore une théorie. $C^{\prime}$ en est un préalable indispensable. Cette vision impose par ailleurs une certaine méthode (ou démarche) pour construire une théorie. 
Cette méthode ne se réduit pas à un ou deux principes généraux. Elle doit être opérationnelle. Le terme d'approche désigne alors le couple constitué par une vision et la méthode accordée à cette vision : la vision retenue impose cette méthode et on ne peut avoir d'autre vision que celle-ci, si on retient cette méthode. Une approche particulière peut présider à l'établissement de nombreuses théories partielles ou même être à l'amont de plusieurs programmes de recherche (au sens de Lakatos). Le domaine en question peut être plus ou moins étendu. La vision comprend toujours une délimitation de ce domaine et par voie de conséquences de l'objet en question. Une approche générale de la science sociale (ou une approche générale en science sociale, comme on voudra) couple donc une vision de la vie des hommes en société et une méthode opérationnelle pour construire un savoir à son sujet. Elle ne se confond pas avec une préoccupation particulière la concernant, comme par exemple celle de comprendre comment les normes sociales émergent de l'interaction des individus. En effet, une préoccupation peut être commune à plusieurs approches - ce qui est le cas, on va le voir, pour la précédente. D'ailleurs, une préoccupation n'est pas non plus le critère distinctif d'une problématique générale en science sociale.

Une problématique déborde une seule approche et peut être ainsi le fond commun de plusieurs approches. Elle repose aussi sur une vision; mais celle-ci est plus large, moins précise. Cette vision s'accorde à un principe méthodologique, dont l'énoncé ne dicte pas une seule façon de procéder pour établir un savoir positif dans le domaine considéré. Il revient au même de dire que l'on ne peut remonter de ce principe à la vision qui le commande. C'est en ce sens que l'on parle en science sociale d'une problématique rationaliste et d'une problématique structuraliste. L'erreur est de penser que la première se comprendrait en partant de l'individualisme méthodologique et la seconde, du holisme méthodologique. La vision relève de la philosophie sociale. C'est la porte d'entrée qui s'impose pour traiter de l'affirmation d'une nouvelle problématique dite institutionnaliste.

\subsection{Les deux versants de la vision d'un comportement individuel: penser/agir}

La philosophie sociale s'entend pour distinguer deux versants de tout comportement d'un individu (ou de tout acte, si on préfère) ${ }^{9}$. Le premier est le versant subjectif tenant à la capacité de l'homme à penser à ce qu'il va faire ou à ce qu'il a fait. Une conception du temps, distincte du temps chronologique, lui est associée, ainsi que les concepts de signification et d'intention (voir tableau 1). L'accès à ce premier versant passe par une

9 Voir notamment [Descombes, 1996].

9 Economie et Institutions $-\mathrm{n}^{\circ} 4-1^{\mathrm{e}}$ semestre 2004 
écoute de ce que dit la personne et le chercheur en rend compte par un exposé à la première personne. Le second est le versant objectif tenant à la capacité de quiconque d'observer de l'extérieur l'agir de tel ou tel et d'en rendre compte par un discours à la troisième personne. Ce versant met en jeu le temps chronologique, les concepts associés étant ceux d'orientation et de finalité (voir tableau 1). On rend bien compte de ces deux versants en disant que «les hommes vivent dans le futur et agissent dans le présent » [Commons, $1934: 84]$.

\section{Tableau 1 : Les deux versants de la vision d'un comportement}

$$
\text { (ou acte) individuel }
$$

\begin{tabular}{|l|l|l|}
\cline { 2 - 3 } \multicolumn{1}{c|}{} & $\begin{array}{l}\text { Le versant du pensé } \\
\text { (subjectif) }\end{array}$ & $\begin{array}{l}\text { Le versant de l'agir } \\
\text { (objectif) }\end{array}$ \\
\hline Le temps pris en compte & $\begin{array}{l}\text { Le temps futur (le déplacement dans } \\
\text { le temps par la pensée à un moment } \\
\text { du temps chronologique)* }\end{array}$ & $\begin{array}{l}\text { Le temps chronologique } \\
\text { (l'acte est observé de l'extérieur } \\
\text { à tel moment de ce temps) }\end{array}$ \\
\hline Les concepts associés & $\begin{array}{l}\text { Signification : le sens que la personne } \\
\text { communique (les raisons qu'elle donne } \\
\text { à ce qu'elle va faire, ou a fait, à qui } \\
\text { lui demande) }\end{array}$ & $\begin{array}{l}\text { Orientation : le sens que retient } \\
\text { l'analyse du chercheur pour } \\
\text { expliquer l'acte observé } \\
\text { (orientation } \\
\text { causale/orientation téléologique }\end{array}$ \\
\cline { 2 - 3 } & $\begin{array}{l}\text { Intention (acte intentionnel) : } \\
\text { la tournure que prend la signification } \\
\text { lorsque celle-ci est un sens } \\
\text { visé (sinon, l'acte est } \\
\text { non intentionnel) }\end{array}$ & $\begin{array}{l}\text { Finalité }: \text { ce que le chercheur retient } \\
\text { comme étant le résultat } \\
\text { de l'agir qui importe } \\
\text { pour insérer ce dernier } \\
\text { dans la vie sociale }\end{array}$ \\
\hline
\end{tabular}

*Peirce [1879] parle à ce propos de futurité.

**L'acte est déterminé par des causes/l'acte est tiré par le désir d'un résultat qui en est attendu [Simmel, $1987: 238$ et suiv.].

Tous courants et disciplines confondus, les chercheurs en science sociale s'accordent pour retenir que la capacité de l'homme à se projeter dans l'avenir par la pensée est une disposition de première nature (elle a été acquise à une époque qui remonte à la nuit des temps ; elle n'est pas propre à telle ou telle sorte de société). Le débat porte d'abord sur la nécessité ou non de prendre en compte le versant "penser» pour saisir le versant "agir»: doit-on prendre en compte la signification pour pouvoir dire quelque chose à propos de l'orientation? Et ensuite, si on juge cela nécessaire, sur la façon dont on doit articuler ces deux versants: peut-on saisir le premier versant, notamment comprendre la signification, sans tenir compte du contexte objectif de l'agir, c'est à dire des institutions de la société dans laquelle vit (dans le futur) l'individu et qui sont déjà là ? 


\subsection{Problématique rationaliste et visions de la rationalité}

La problématique rationaliste (PR) est celle qui voit la rationalité à l'amont des comportements des individus, en retenant que celle-ci est de première nature. Elle consiste donc (i) à postuler que tout acte relève d'une analyse à orientation téléologique - l'acte n'est pas déterminé par des causes ; il est tiré par les effets qui en sont attendus, (ii) à considérer que l'on $\mathrm{n}$ 'a pas besoin de prendre en compte la signification du comportement pour déterminer l'effet visé par l'auteur de l'acte - cet effet est la satisfaction de son propre intérêt exprimé par une fonction agrégeant des désirs (par exemple, les satisfactions apportées par des biens, s'agissant de la rationalité de l'homo œeconomicus) et (iii) que l'on n'a pas non plus besoin de tenir compte de la société dans laquelle vit l'individu pour délimiter ces désirs. Cet acte est un choix (ou une décision).

La représentation rationaliste d'un comportement rationnel ne se réduit pas à celle de l'approche rationaliste standard

Il est important de noter que cette représentation d'un comportement rationnel ne se réduit pas à celle qui est constitutive de l'approche rationaliste standard. Dans cette approche particulière qui est, on va le voir sous peu, la principale approche relevant de la PR, on ne voit aucune règle à l'amont des comportements individuels, ce qui va de pair avec le fait qu'un acteur n'a pas à s'interroger sur les comportements présents ou à venir des autres acteurs avant toute action de sa part. L'agrégation des comportements est alors vue comme une simple sommation sans interaction (dite stratégique, en théorie des jeux). La rationalité dont est doté l'individu est à même d'opérer sans règles. Des croyances, qui lui sont propres, lui suffisent. Les comportements relèvent donc d'une perspective strictement calculatrice, sans rien de culturel.

Au contraire, cette représentation peut laisser place à la présence de règles contraignant les actes. Il n'en reste pas moins que ces règles sont librement choisies par des individus rationnels, étant donné le contexte dans lequel ils doivent se coordonner. Pour le dire autrement, on ne voit pas d'objets collectifs à l'amont de la rationalité. Doit-on considérer que cette représentation d'un comportement rationnel est la seule possible?

11 Economie et Institutions $-\mathrm{n}^{\circ} 4-1^{\mathrm{e}}$ semestre 2004 
Une déclinaison de la rationalité autorisant qu'elle soit de seconde nature: l'hypothèse d'une formation sociale de la rationalité

La représentation n'est plus la même si on adopte le point de vue de Max Weber [1995]. Pour ce dernier, on doit se reporter à la signification pour comprendre ce qu'est un comportement rationnel : un comportement peut être à signification émotionnelle, traditionnelle, rationnelle en valeur ou rationnelle en finalité. Un réexamen critique de cet apport conduit à remarquer 1/ que Weber n'explicite pas le fond commun de la rationalité en valeur et de la rationalité en finalité et $2 /$ qu'il ne distingue pas un acte d'une activité. Par activité, on entend toute façon pour un être humain d'occuper son temps en un lieu donné, caractérisé par un certain environnement, en mobilisant des ressources et en étant capable, si on lui demande, de communiquer un sens subjectif à cette activité. Cette dernière comprend le plus souvent une succession d'actes ou de comportements ${ }^{10}$. Seul un acte (comportement) peut être à signification émotionnelle. A la différence d'un acte émotionnel, une activité est intentionnelle: le sens communiqué est un sens visé. On ne peut parler de signification traditionnelle ou rationnelle que pour une activité ${ }^{11}$. A une certaine étape du processus d'individuation dans l'histoire, le sens subjectif communiqué par l'individu qui s'active se réfère essentiellement, non plus à la tradition, mais à ses propres besoins/désirs (elle lui permet d'en satisfaire un ou plusieurs). Cette activité devient pour lui une activité rationnelle (en valeur et/ou en finalité). La rationalité est donc conçue comme une disposition acquise à une certaine étape de l'histoire humaine. Ce n'est pas une propriété de première nature ${ }^{12}$. Autrement dit, cette disposition ne s'applique qu'à des contextes sociaux dans lesquels l'individu a une existence telle qu'il lui est possible de

10 On peut ajouter que cette activité a des effets ou résultats. Sa finalité est l'un de ces effets. C'est l'effet essentiellement visé par l'agent qui s'y livre ou par celui qui la commande lorsqu'elle est menée en situation de subordination. Cette définition est une appropriation de celle de Weber pour qui une activité est " tout comportement humain (peu importe qu'il s'agisse d'un acte extérieur ou intime, d'une omission ou d'une tolérance) quand et pour autant que l'agent ou les agents lui communiquent un sens subjectif " [1995:28].

11 Plus précisément, on ne peut le dire d'un comportement qu'en tant qu'il participe à la réalisation d'une activité.

12 La définition retenue (voir tableau 3) implique d'avoir recours à la disposition de Pierce et à la compétence de Giddens. Pour Charles Peirce, une disposition est une inclination à agir dans un sens déterminé acquise par inférence hypothétique, c'est-à-dire par l'ajustement progressif d'hypothèses explicatives à une série d'expériences singulières [Bourdieu, 1998], [Servais, 2002]. Pour Anthony Giddens, cette knowledgebility tient au fait que " une personne est un agent qui se donne des buts, qui a des raisons de faire ce qu'il fait et qui est capable, si on lui demande, d'exprimer ces raisons de façon discursive " [1987: 51].

Economie et Institutions $-\mathrm{n}^{\circ} 4-1^{\mathrm{e}}$ semestre 2004 
parler de son propre intérêt ${ }^{13}$. Cela vaut, en particulier, pour la société moderne à laquelle on s'en tient dans la suite. A partir de ce moment, l'institution des règles du jeu social est précédée d'un débat. Ce dernier donne lieu à l'expression de critiques et de justifications (voir tableau 2). Ces discours se distinguent alors nettement des significations qui sont exprimées dans le cours du jeu social, dans la mesure où ils portent explicitement sur les règles devant habiliter, contraindre ou interdire telle ou telle pratique.

Tableau 2 : Les justifications

\begin{tabular}{|c|c|c|c|}
\hline & $\begin{array}{l}\text { Justification } \\
\text { individuelle }\end{array}$ & \multicolumn{2}{|l|}{ Justification sociale } \\
\hline $\begin{array}{l}\text { Savante } \\
\text { (exprimée } \\
\text { par un chercheur } \\
\text { en science sociale) }\end{array}$ & & \multicolumn{2}{|c|}{$\begin{array}{l}\text { Le chercheur démontre que telle } \\
\text { règle (ou institution) conduit bien } \\
\text { à la satisfaction d'un objectif social } \\
\text { fixé a priori (voir AIR, infra) }\end{array}$} \\
\hline \multirow[t]{2}{*}{$\begin{array}{l}\text { Ordinaire (exprimée } \\
\text { par l'homme } \\
\text { de la rue - le citoyen) }\end{array}$} & \multirow[t]{2}{*}{$\begin{array}{l}\text { L'individu exprime son } \\
\text { intérêt propre à ce que } \\
\text { telle règle soit instituée }\end{array}$} & \multicolumn{2}{|c|}{$\begin{array}{l}\text { L'individu argumente en faveur de telle règle } \\
\text { (institution) en mettant en avant } \\
\text { contribution à l'ordre social }\end{array}$} \\
\hline & & $\begin{array}{l}\text { Justification sociale } \\
\text { commune: } \\
\text { tous les membres de } \\
\text { la société finissent } \\
\text { par s'entendre sur } \\
\text { la même justification } \\
\text { (voir AISo, infra) }\end{array}$ & $\begin{array}{l}\text { Justification sociale } \\
\text { strictement collective: } \\
\text { justification en droit } \\
\text { (voir AIH, infra) }\end{array}$ \\
\hline
\end{tabular}

L'hypothèse est alors qu'on est en présence d'une formation sociale de la rationalité, c'est à dire d'une formation sociale des préférences de l'individu, de ses croyances, des valeurs auxquelles il se réfère, ainsi que des représentations qu'il se fait des conséquences de ses actes. Cette conception générale de la rationalité s'oppose en cela à celle de la PR. Au cours des vingt dernières années, diverses tentatives de raccordement entre ces deux « entrées » dans la rationalité ont été proposées, d'un côté en déclinant la

13 Dans cette définition de la rationalité " en général ", les critères retenus par l'individu pour apprécier s'il agit dans son propre intérêt ne sont pas du tout spécifiés ; ses désirs ne sont pas actualisés dans une liste particulière. Par désir, j'entends le résultat d'une double conversion [Scikovsky, 1978], la conversion d'un affect en l'expression du besoin de quelque chose (orientation causale) et celle du besoin en désir (orientation téléologique), sous l'effet du conatus, qui est la propension de tout être humain à persévérer dans l'être, l'impulsion de l'action de l'être humain selon Spinoza [Lordon, 2003]. L'hypothèse faite est alors que, même quand ils sont présentés comme tels par celui qui les exprime, les désirs d'un individu ne lui sont pas propres ; chacun désire ce que l'autre désire [Elias, 1985], [Girard, 1978].

13 Economie et Institutions $-\mathrm{n}^{\circ} 4-1^{\mathrm{e}}$ semestre 2004 
rationalité en finalité de Weber [Boudon, 1989], de l'autre en remontant à partir de la rationalité substantielle parfaite de Savage, comme le fait Simon [1976] en critiquant son caractère irréaliste. Une solution de synthèse possible est celle qui est présentée dans la figure 1, avec les définitions données dans le tableau 3 annexé à cette figure ${ }^{14}$. Cette déclinaison permet de voir que l'on ne peut abandonner l'hypothèse d'une formation sociale de la rationalité que lorsqu'on passe à la rationalité instrumentale et que cette dernière n'est à même d'opérer sans règles que lorsqu'elle est substantielleparfaite. A l'amont de la rationalité instrumentale, on doit ressaisir les justifications et les significations pour comprendre la rationalité.

Figure 1: Le développement du concept de rationalie

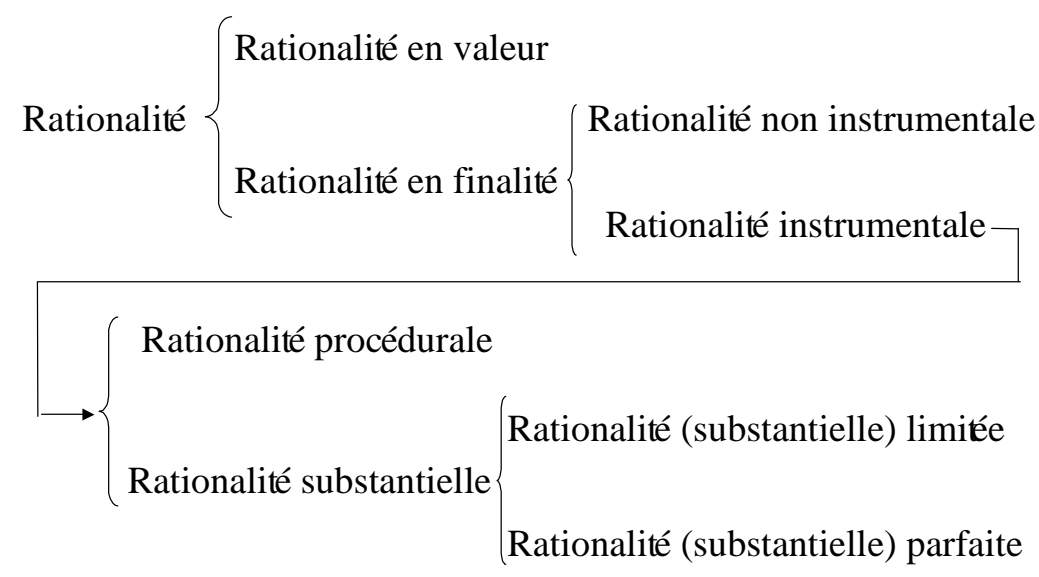

14 Précisons que les formes qui sont déclinées sont des formes polaires, idéales typiques au sens de Weber, et non des types distincts ne pouvant se combiner dans les pratiques concrètes.

Economie et Institutions $-n^{\circ} 4-1^{e}$ semestre 2004 

typiques

Rationalité (tout court) : attribut d'un individu doté d'une disposition (Peirce), celle d'agir dans son propre intérêt. Cela signifie que, si on lui demande, il est capable (Giddens) de donner à chacune de ses activités un sens subjectif (Weber) qui fait référence à ses propres désirs, sans exclure alors l'altruisme - désirer satisfaire le désir de l'autre - et $a$ fortiori l'attention à l'autre. Une activité à signification rationnelle n'est donc pas nécessairement contrôlée de façon réflexive et $a$ fortiori précédée d'un raisonnement. La communication du sens visé peut se faire a posteriori (la conscience pratique de Giddens).

Rationalité (absolue) en valeur : l'individu agit de façon rationnelle "sans tenir compte des conséquences prévisibles de ses actes, au service qu'il est de sa conviction portant sur ce qui lui apparaît commandé par le devoir, la dignité, la beauté, les directives religieuses ou la grandeur d'une cause, quelle qu'en soit la nature » [Weber, $1995: 56]$. L'individu croit à la valeur intrinsèque - d'ordre éthique, esthétique, religieux ou autre - de son activité.

Rationalité (absolue) en finalité : l'individu agit de façon rationnelle « en tenant compte des conséquences prévisibles de ses actes et en adaptant au mieux les moyens disponibles à la fin propre visée » [Weber, $1995: 57]$.

Rationalité instrumentale : agir de façon rationnelle en finalité dans un contexte où « la fin et les moyens de l'action sont parfaitement séparés» et en ne faisant intervenir "aucune considération morale ou politique concernant la validité des moyens eux-mêmes » [Postel, 2003 : 29]. L'individu est ainsi doté d'une nouvelle disposition, celle de réfléchir avant toute pratique : cette dernière est (ou résulte de) une décision, c'est-à-dire un choix entre diverses solutions. La rationalité devient alors utilitariste: les désirs se portent sur des biens jugés utiles, y compris biens communs. On exclut alors à la fois le désir de satisfaire le désir de l'autre et celui de désirer sa présence (communiquer avec lui). Un bien est un objet que l'on désire. L'objectif de l'individu est de disposer de biens qui lui apportent de la satisfaction. L'autre devient un instrument au service de cet objectif.

Rationalité (instrumentale) procédurale : la décision est un processus cognitif qui se déroule dans un contexte d'incertitude radicale. L'individu n'est pas dans un contexte tel qu'il aurait à choisir entre des solutions déjà là (comme on tire une boule dans un sac qui en contient déjà) ; il construit progressivement la solution qu'il va retenir. L'individu doit porter attention à l'autre: que va-t-il faire en réaction à ma propre décision? La rationalité de la décision finalement prise (en termes d'intérêt propre) est appréciée au regard de la procédure suivie [Simon, 1976]. La procédure en question se définit par des règles, de type conventions.

Rationalité (instrumentale) substantielle : la décision est prise au regard de l'objectif poursuivi, objectif qui est accessible en principe [Simon, 1976]. Cela nécessite le recours à des croyances pour surmonter l'incertitude radicale. L'individu calcule ce que lui rapporte chacun des choix possibles (en les classant selon ce rapport). Ce rapport est le critère retenu pour décider du bon choix. Un choix est préféré à un autre s'il rapporte plus. Comme cette rationalité est utilitariste, ce rapport est exprimé en utilité apportée par la consommation d'un panier de biens (hypothèse d'une fonction d'utilité).

Rationalité substantielle limitée : l'individu rencontre des limites dans la mise en œuvre de ce mode de choix substantiellement rationnel, en raison de capacités cognitives limitées (capacité de calcul limitée, manque d'informations,...). L'individu ne calcule que le rapport (absolu ou relatif) de certains choix. Il s'arrête lorsqu'il juge le rapport satisfaisant (March et Simon, première version).

Rationalité substantielle parfaite : la rationalité substantielle opère parfaitement. L'individu dispose d'une information complète sur la structure du problème auquel il est confronté, ainsi que d'une capacité de calcul infinie sans coûts et d'un ensemble complet et ordonné de préférences (Savage).

15 Economie et Institutions $-\mathrm{n}^{\circ} 4-1^{\mathrm{e}}$ semestre 2004 
La PR ne se limite pas à la vision de la rationalité comme étant substantielle. On sort de la PR lorsqu'on ne voit plus l'individu comme quelqu'un qui est totalement maître et libre de ses propres désirs - ces derniers lui seraient propres au sens fort du terme. On passe à une autre problématique lorsqu'on voit la rationalité comme un produit de la société dans laquelle vit l'individu ou encore comme une règle sociale. Pour autant, ce n'est pas parce qu'on voit la rationalité comme étant endogène à la société que l'on doit nécessairement se préoccuper de développer une théorie dont l'objet est de rendre compte de cette formation sociale des préférences et des croyances des individus. Il est tout à fait possible, comme le fait J. S. Coleman avec sa théorie du choix rationnel, de construire une théorie dans laquelle on se donne au départ la rationalité déjà formée parce que l'objectif scientifique que l'on poursuit est seulement d'en analyser les implications, une telle théorie relevant d'une méthodologie dans laquelle la rationalité est prise comme exogène. Autrement dit, il y a place pour une approche à rationalité exogène en termes de méthode au sein d'une problématique à rationalité endogène en termes de vision.

\subsection{Problématique structuraliste et visions holistes}

Il n'y a aucune symétrie entre la problématique structuraliste (PS) et la PR. Présente dans toutes les sciences sociales, notamment avec le marxisme, la PS est d'emblée plurielle, avec une vive lutte interne à ce champ quand à la "bonne» façon d'être structuraliste [Théret, 2003]. Beaucoup, notamment parmi ceux qui critiquent cette problématique, entendent la réduire à la seule version standard dans laquelle la vision est de ne voir que des règles à l'amont des comportements des individus sans rien qui tiendrait à eux, c'est à dire à s'en tenir à une stricte orientation causale sans juger nécessaire de se préoccuper de la signification. Le propre de la PS est seulement d'adopter une vision holiste de la vie sociale. On doit la qualifier de vision holiste-structuraliste, ou encore faire état à son propos d'un holisme structural, parce qu'il y a place pour une autre version de la vision holiste, celle que Vincent Descombes [1996] appelle le holisme collectiviste et que je préfère qualifier de holisme ensembliste.

\section{La vision holiste-structuraliste : le holisme structural}

La vision holiste de la vie sociale s'oppose à la vision individualiste, dans laquelle les individus sont regardés comme des acteurs indépendants faisant des choix dans des situations définies par des ressources et des contraintes et dans laquelle, en conséquence, on voit les structures collectives (ou encore les objets collectifs) comme de simples effets émergeant de la composition des actions individuelles. Le point de vue holiste est au contraire que le cadre institutionnel est un cadre collectif qui n'est

Economie et Institutions $-n^{\circ} 4-1^{e}$ semestre 2004 
pas influençable par un individu isolé. On aura l'occasion de préciser dans la suite que, à ce titre, la vision holiste ne peut s'accorder qu'avec l'hypothèse méthodologique $\mathrm{d}^{\prime}$ une rationalité endogène. La question qui nous occupe à cette étape est seulement de savoir si la vision holiste est nécessairement structuraliste (en un sens à préciser) ou si elle comprend aussi une version faible.

La vision holiste-structuraliste en science sociale est la simple application en ce domaine de la façon holiste-structuraliste tout à fait générale de voir un objet, que ce dernier soit un objet matériel, un individu ou la vie sociale. Cet objet se compose d'éléments; ces éléments sont vus à la fois comme étant en relation entre eux et comme faisant partie d'un tout; en conséquence, " on ne peut penser ces éléments qu'en relation entre eux et qu'en tant que partie d'un tout» [Théret, 2003: 16], c'est-à-dire «en s'interdisant toute autre hypothèse sur les éléments considérés (en particulier toute hypothèse sur leur «nature » propre) » [Descombes, 1979: 104]. Cela revient à voir cet objet comme un système et à retenir que «les relations qui sont au fondement du système» en sont la structure [Descombes, $1996: 156]^{15}$.

En science sociale, les éléments sont les individus et l'objet, la "société» qu'ils forment (cette société peut être une organisation particulière - famille, entreprise, association, réseau - ou une société territorialisée - locale, nationale ou mondiale). Le structuraliste voit cette « société » comme une structure, qui peut en comprendre d'autres et/ou être elle-même emboîtée dans d'autres. Cela implique qu'il ne fait au départ aucune hypothèse sur une première nature de l'homme, une première nature qu'il pourrait qualifier sans prendre en compte la société dans laquelle vit l'individu. Reste qu'on ne voit pas la structure, parce que celle-ci est une construction de l'esprit; seuls des modèles de cette structure ont une existence (on y revient dans la suite). Ce qu'il voit à l'amont des comportements des individus, ce sont les règles par l'intermédiaire desquelles la structure agit les individus, c'est-à-dire détermine, façonne, canalise ou guide leurs comportements. Par le fait même, ces derniers ont la propriété de reproduire la structure, quitte à ce que cette reproduction passe par un changement des modèles dans lesquels elle s'actualisait. Le structuraliste voit des règles à l'amont de ces comportements.

15 Cette définition très générale englobe celle de Jean Piaget, pour qui une structure est " un système de transformation, qui comporte des lois en tant que systèmes (par opposition aux propriétés des éléments), et qui se conserve ou s'enrichit par le jeu même de ses transformations, sans que celles-ci aboutissent en dehors de ses frontières ou fasse appel à des éléments extérieurs. En un mot, une structure comprend ainsi les trois caractères de totalité, de transformation et d'autoréglage " [1968: 6-7].

17 Economie et Institutions - n ${ }^{\circ} 4-1^{\text {e }}$ semestre 2004 
Cela n'interdit pas de faire une place à l'individu ${ }^{16}$. Ceux qui se préoccupent de comprendre comment et pourquoi s'effectue une adhésion de l'individu à des règles mettent l'accent sur le côté informel de ce processus de socialisation de l'individu, c'est-à-dire sur l'incorporation de la structure, sur la structuration de l'individu au sein de la société - l'habitus de P. Bourdieu, la conscience pratique de Giddens ${ }^{17}$. Il y est question d'intentionnalité, notamment chez Commons [1934], de motivations. D'ailleurs, les règles ne sont pas la seule médiation entre la structure et le comportement individuel. Ces autres médiations sont les valeurs, les représentations, qui pour être sociales n'en relèvent pas moins pour certains chercheurs de la structuration de l'individu. Cela revient à considérer que les raisons pour lesquelles les règles sont suivies - ou ne le sont pas en cas de déstructuration - ont au moins quelque chose à voir avec la justice, sans exclure l'intérêt propre. Et cela conduit à ne pas réduire ou identifier la vision holiste-structuraliste à celle du seul structuro-fonctionnalisme consistant à « ramener tous les systèmes d'interaction à de simples systèmes de rôles » [Boudon, 1979: 89].

Une version faible de la vision holiste : le holisme ensembliste

L'un des apports de Vincent Descombes [1996] est de nous inviter à ne pas commettre une seconde réduction, celle qui consiste à ramener la vision holiste à la seule vision holiste-structuraliste qui vient d'être caractérisée. Deux holismes sont en effet distingués par lui : le "holisme structural » et le «holisme collectiviste». A la différence du premier, le second « ne connaît pas de structures » parce qu'il « ne connaît de totalité ou d'ensemble que sous la forme d'une collection d'individus, c'est-à-dire d'une "réunion physique ou mentale d'éléments » simples, sans autre principe de composition entre eux que celui d'une commune appartenance à l'ensemble sur la base d'une identité individuelle partagée $»^{18}$. Pour des raisons que l'on

16 Ainsi, Etienne Balibar [1997] l'envisage comme un sujet. Pour sa part, Claude Levi-Strauss considère que "nous ne pouvons jamais être sûrs d'avoir atteint le sens et la fonction d'une institution, si nous ne sommes pas en mesure de revivre son incidence sur une conscience individuelle. Comme cette incidence est une partie intégrante de l'institution, toute interprétation doit faire coïncider l'objectivité de l'analyse historique ou comparative avec la subjectivité de l'expérience vécue " [1991: XXVI] (cité par [Theret, 2003]).

17 Pierre Bourdieu retient que l'adhésion aux règles serait " l'effet historique de l'accord entre deux réalisations du social, dans les choses par l'institution et dans les corps par l'incorporation (habitus)" [1980]. Pour sa part, Anthony Giddens comprend cette "incorporation ", en concevant la structuration de l'individu comme l'articulation entre l'inconscient, la conscience pratique et la conscience discursive. On trouve dans [Lipietz, 1988] une tentative de synthèse entre ces deux auteurs.

18 [Theret, $2003: 7$ ] résumant en le citant [Descombes, $1996: 138$ ].

Economie et Institutions $-n^{\circ} 4-1^{e}$ semestre 2004 
comprendra mieux dans la suite, je préfère parler de holisme ensembliste ou encore de holisme du commun. Dans cette forme faible de vision holiste, on ne fait pas de place aux relations entre les éléments du système. On sort de la PS lorsqu' on adopte une telle vision holiste-ensembliste.

\subsection{Une première identification en compréhension de la problématique institutionnaliste}

La PR et la PS sont deux problématiques tout à fait extérieures l'une à l'autre, ne serait qu'en raison du fait que le holisme ensembliste ne trouve place ni dans l'une ni dans l'autre. On ne peut toutefois associer la problématique institutionnaliste (PI), que l'on cherche à identifier en compréhension, à ce critère distinctif intermédiaire entre la PR et la PS. Il est trop réducteur (on va voir qu'il est constitutif de l'une des approches qui se situent dans la PI $)^{19}$. L'hypothèse qui s'impose est donc que la PI recouvre partiellement à la fois la PR et la PS. Si elle est dotée d'une consistance propre, celle-ci doit se présenter comme une combinaison appropriée de leurs visions respectives. L'enjeu est de surmonter l'opposition traditionnelle entre deux représentations de l'homme, celle de l'homo oeconomicus agissant de façon rationnelle et celle de l'homo sociologicus mu par des normes sociales [Orlean, 1994]. Une telle combinaison est-elle possible?

\section{Une combinaison appropriée de la vision de la PR et de la vision de la PS}

Les deux visions à combiner sont de plus en plus considérées dans le champ qui nous occupe comme deux «réponses à la dimension la plus importante du problème de la structure et de l'agent, à savoir : comment on peut dire d'une institution qu'elle structure l'action humaine, dans un sens plus ou moins déterministe, de façon à produire un modèle normalisé de comportement, alors que l'existence de l'institution elle-même dépend habituellement de la présence de ces modèles de comportement et, par conséquent, de la disposition des acteurs à se comporter d'une certaine façon. Le problème consiste à exprimer simultanément le caractère volontaire et déterministe de ces institutions » [Hall, Taylor, 1997 : 473]. Ce que l'une des deux visions en question donne à voir, l'autre ne le voit pas. Combiner les deux est la solution. Mais comme ces deux visions sont contradictoires, cette combinaison implique de modifier quelque peu l'une et l'autre.

19 L'entité ainsi délimitée ne comprend ni l'ancien institutionnalisme de Commons, qui relève du holisme structural, ni le nouvel institutionnalisme de Williamson, qui s'en tient à une vision individualiste sans pour autant relever de la PR (voir notamment [1996]).

19 Economie et Institutions $-\mathrm{n}^{\circ} 4-1^{\mathrm{e}}$ semestre 2004 
On doit d'abord ouvrir la vision de la PR à la possibilité que des règles puissent être vues, en plus de la rationalité, à l'amont des comportements. Le relâchement opéré exige que ces règles soient regardées comme un effet de composition des comportements individuels. De ce fait, on ne peut plus s'en tenir à une rationalité substantielle parfaite. On doit au moins remonter à la rationalité limitée. Ce que l'on a ainsi ajouté à l'amont des comportements s'accorde à la vision de la PS. Il n'en reste pas moins que l'on doit procéder à une double révision de cette dernière. La première révision la complexifie : tous les objets collectifs doivent être regardés, non plus comme donnés au départ, mais comme endogènes aux comportements individuels. La seconde est un relâchement équivalent à celui auquel on a dû procéder pour la vision de la PR: on doit ouvrir la possibilité que la rationalité puisse être vue, en plus de règles, à l'amont des comportements.

\section{L'identité propre de la PI}

Le signe distinctif de la problématique institutionnaliste ainsi construite en termes de vision est donc de voir à la fois des règles et la rationalité à l'amont des comportements individuels (voir figure 2).

Figure 2 : PR, PS et PI : un recouvrement partiel

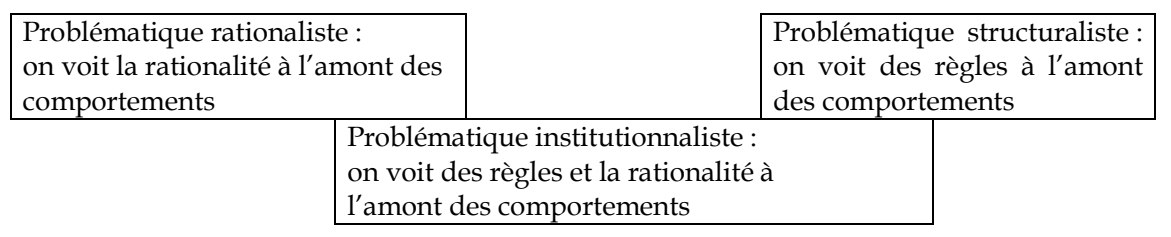

Plus précisément, la PI consiste à regarder les phénomènes sociaux comme le résultat de l'interaction de comportements individuels conformes à des règles que les individus jugent rationnel de suivre en les interprétant s'il y a lieu. D'une situation ou d'un contexte d'interaction à l'autre, ces règles changent. Il s'agit à chaque fois d'un système de règles, c'est à dire d'une institution (sociale) conçue comme un objet collectif dont les valeurs sont exclues. En effet, on ne considère plus simplement celles-ci comme des principes généraux à la lumière desquels les prescriptions et les interdits peuvent être légitimés par l'individu, mais comme «des conceptions implicites ou explicitent du désirable qui influencent les choix parmi les modes, moyens et fins possibles de l'action » [Klockhohn, 1951] et qui sont à ce titre incorporées dans les individus. Pour le dire autrement, les comportements concilient la justice sociale et l'intérêt propre, la première se traduisant par des règles et le second pouvant mettre en jeu des valeurs et 
des représentations. Quand à la rationalité, dont chaque individu est doté et qui permet l'interprétation des règles, la double appropriation opérée n'impose pas de retenir qu'elle serait de seconde nature, c'est à dire de retenir l'hypothèse d'une formation sociale des préférences, des croyances et des valeurs. A ce titre, on est en présence d'une problématique ouverte à une pluralité de visions plus précises.

Ce premier cadrage de la PI ne permet pas de statuer sur les contenus respectifs des deux recouvrements partiels visualisés dans la figure 2, celui de la PI par la PR d'un côté, celui de la PI par la PS de l'autre. On ne peut y parvenir qu'en couplant vision et méthode. On verra qu'il n'y a aucune symétrie entre ces deux recouvrements.

\section{Trois approches institutionnalistes distinctes}

L'entrée dans cette seconde partie est strictement méthodologique. On construit les positionnements méthodologiques possibles en science sociale à partir de l'état actuel du débat entre l'individualisme méthodologique et le holisme méthodologique. Il y en a cinq. Trois de ces normes s'accordent à la PI. On présente alors les trois approches « institutionnalistes » qui correspondent à ces trois normes méthodologiques (il s'agit d'un premier cadrage qui sera complété dans la troisième partie).

\subsection{A partir de l'état actuel du débat entre l'individualisme méthodologique et le holisme méthodologique: cinq normes méthodologiques possibles en science sociale}

Une approche est souvent réduite à sa seconde composante, la méthode d'analyse ou méthodologie mise en œuvre. La principale raison en est que les différences entre approches d'un même objet ne ressortent clairement - elles ne peuvent être objectivement explicitées - qu'au plan de la méthode. La méthode est donc la bonne porte d'entrée pour distinguer diverses approches générales en science sociale, notamment au sein de la PI.

2.2. Les termes actuels du débat entre l'individualisme méthodologique et le holisme méthodologique : un espace de recouvrement et une absence de symétrie

Commun à toutes les sciences sociales, le débat méthodologique traditionnel et récurrent est celui qui oppose l'individualisme méthodologique (IM) et le holisme méthodologique (HM). Ce débat est relatif à la démarche que l'on doit retenir pour construire une théorie

21 Economie et Institutions $-\mathrm{n}^{\circ} 4-1^{\mathrm{e}}$ semestre 2004 
explicative ou compréhensive des phénomènes sociaux. De quoi doit-on partir ? Par quoi doit-on passer? La question posée est de savoir comment sont pris en compte les comportements individuels et les objets collectifs dans le cours de cette démarche.

La vulgate en la matière est de considérer que l'on est en présence de deux principes méthodologiques strictement opposés, l'un étant en quelque sorte le symétrique de l'autre. Puisqu'on doit en tout état de cause partir de quelque chose pour construire une théorie, l'individualisme méthodologique (IM) dirait que " on doit partir des individus", tandis que le holisme méthodologique (HM) dirait que «on doit partir d'objets collectifs». Ce n'est pas aussi simple. Voyons d'abord ce qu'il en est précisément pour $1^{\prime} \mathrm{IM}^{20}$. Selon la définition qu'en donne Mark Blaug, le principe de l'IM « affirme que l'on ne peut considérer comme adéquates les explications des phénomènes sociaux, politiques et économiques que si elles sont formulées en termes de croyances, d'attitudes et de décisions individuelles » [Blaug, $1994: 44]$. Ce principe ne dit pas que « on doit partir des individus ». Il ne s'agit là que d'une modalité simple de mise en œuvre de ce principe, qui peut être qualifiée d'IM simple. Ce principe dit seulement que «on doit passer par les individus». Le point de départ peut donc tout aussi bien être en tout ou partie relatif à des objets collectifs, à condition que l'on passe par les individus, c'est-à-dire que l'on ressaisisse leurs comportements dans le cours de la démarche. Il s'agit alors d'un IM complexe. De plus, l'IM simple et l'IM complexe se décomposent eux aussi (voir figure 3 et le tableau 4 annexé).

Le principe de l'HM est une application en science sociale de la méthode holiste d'analyse de tout système d'éléments reliés entre eux et formant un tout. Ce principe est que " les ensembles sociaux sont censés avoir des finalités et des fonctions qui ne peuvent pas être réduites aux croyances, attitudes et actions des individus qui les composent» [Blaug, 1994 : 44]. Ce principe énonce seulement un rejet. Il ne dit pas positivement que « on doit partir des objets collectifs ». Cette expression positive en est la formulation normale, l'HM normal. Le principe général dit seulement que " on ne doit pas partir des individus ». En conséquence, il n'impose pas le contraire de l'IM, puisque cette proposition contraire est que " on ne doit pas passer par les individus». A la limite d'ailleurs, aucun de ceux qui retiennent la méthode holiste ne défendent un tel interdit. Le débat porte sur la façon de passer par les individus. Il y a en fin de compte trois modalités

20Je m'appuie sur les réexamens de l'IM réalisés par R. Boudon [1985] et J. P. Dupuy [1992], en prenant par ailleurs en compte la critique du premier par C. Mouchot [1996] à propos de la question soulevée par la présence de causalités circulaires (ou croisées, si on préfère).

Economie et Institutions $-n^{\circ} 4-1^{e}$ semestre 2004 
opérationnelles de mise en œuvre de l'HM (voir figure 3 et le tableau 4 annexé).

On est donc conduit à deux conclusions: il y a un espace de recouvrement entre l'IM et l'HM et il n'y a pas de symétrie entre les deux côtés (voir figure 3). Le recouvrement partiel de l'HM par l'IM est l'IM complexe ; quand à l'IM simple et l'HM normal que la vulgate oppose, ils n'ont pas de frontière commune; un fossé les sépare; ce dernier délimite une solution intermédiaire, qui ne fait partie ni de l'un ni de l'autre : c'est $l^{\prime} \mathrm{HM}$ faible. L'absence de symétrie résulte du fait que le principe de l'HM est un énoncé négatif.

Figure 3 : Individualisme méthodologique / Holisme

méthodologique

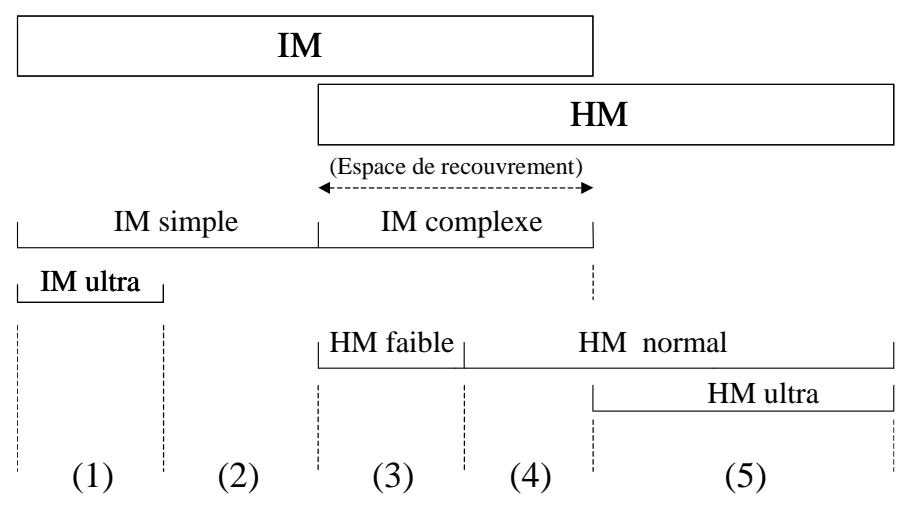

23 Economie et Institutions $-\mathrm{n}^{\circ} 4-1^{\mathrm{e}}$ semestre 2004 
Tableau 4 (annexé à la figure 3) :_Les déclinaisons de l'IM et de l'HM

\begin{tabular}{|c|c|c|c|}
\hline \multirow[t]{4}{*}{$\begin{array}{l}\text { IM } \\
\text { «On doit passer } \\
\text { par les individus » }\end{array}$} & \multirow[t]{2}{*}{$\begin{array}{l}\text { IM simple } \\
\text { «On doit partir des individus ». }\end{array}$} & $\begin{array}{l}\text { IM ultra (1) } \\
\ldots \text { et « on ne doit pas passer } \\
\text { par des objets collectifs » }\end{array}$ & \\
\hline & & $\begin{array}{l}\text { (2) ...et « on doit passer par } \\
\text { des objets collectifs" }\end{array}$ & \\
\hline & \multirow{2}{*}{$\begin{array}{l}\text { IM complexe } \\
\text { "On ne doit pas nécessairement } \\
\text { partir des individus, mais on } \\
\text { doit passer par les individus " }\end{array}$} & $\begin{array}{l}\text { (3) «On part à la fois des } \\
\text { individus et d'objets } \\
\text { collectifs" }\end{array}$ & \multirow[t]{2}{*}{ HM } \\
\hline & & $\begin{array}{l}\text { (4) «On part d'objets collectifs } \\
\text { et on doit passer par } \\
\text { les individus » }\end{array}$ & \\
\hline
\end{tabular}

\begin{tabular}{|c|c|c|}
\hline \multirow{3}{*}{$\begin{array}{l}\text { HM } \\
\text { «On ne doit pas } \\
\text { partir des individus » }\end{array}$} & \multicolumn{2}{|c|}{$\begin{array}{l}\text { HM faible (3) (voir le correspondant en IM) } \\
\text { «On ne part pas seulement d'objets collectifs » }\end{array}$} \\
\hline & \multirow[t]{2}{*}{$\begin{array}{l}\text { HM normal } \\
\text { «On doit partir d'objets collectifs » }\end{array}$} & $\begin{array}{l}\text { (4) (voir le correspondant en IM) } \\
\text {...et « on doit passer } \\
\text { explicitement par les } \\
\text { individus » }\end{array}$ \\
\hline & & $\begin{array}{l}\text { HM ultra (5) } \\
\ldots \text {.et «il n'est pas nécessaire } \\
\text { de passer explicitement } \\
\text { par les individus » }\end{array}$ \\
\hline
\end{tabular}

\section{Conclusion : cinq normes méthodologiques, dont trois permettent de ressaisir les institutions}

Il en résulte qu'on a une palette de méthodes a priori possibles. Ces postures méthodologiques distinctes, ces normes si on préfère, sont au nombre de cinq. Pourquoi choisir telle ou telle? La réponse ne se trouve pas du côté méthodologique. Une méthode n'est rien sans la vision de la vie des hommes en société qui l'accompagne en la justifiant. Seule une vision apporte une réponse à cette question, en donnant par ailleurs le sens précis des expressions utilisées pour qualifier telle ou telle solution de méthode (par exemple, que signifie « passer par les individus » ?).

\section{A ce stade, on peut seulement faire trois constats :}

- l'IM ultra est la seule norme qui ne fait pas explicitement une place aux institutions (les objets collectifs) dans l'analyse ; cette norme est celle de l'approche rationaliste standard définie supra en termes de vision.

- l'HM ultra est celle dans laquelle cette place faite aux institutions se trouve à l'entrée et non dans le cours ou à la sortie de l'analyse ;

Economie et Institutions $-n^{\circ} 4-1^{\text {e }}$ semestre 2004 
cette norme est celle de l'approche structuraliste standard définie supra.

- Les trois normes intermédiaires sont celles dans lesquelles cette place faite aux institutions va de pair avec la nécessité d'analyser tout effet sociétal (phénomène social ou objet collectif) comme le résultat de l'agrégation des comportements individuels dictés par des motivations préalablement ressaisies. Ces trois normes intermédiaires s'accordent à la PI : elles permettent d'analyser la formation des institutions, les différences entre elles laissant entendre que $\mathrm{l}^{\prime}$ « agrégation » en question n'a pas le même sens dans les trois.

On a ainsi une confirmation de la typologie construite par Hall, Taylor et Théret puisqu'on dégage trois méthodes institutionnalistes distinctes, et seulement trois. L'hypothèse que l'on formule est que ces trois normes méthodologiques intermédiaires sont, dans l'ordre, celles qui sont constitutives de l'IR, de l'ISo et de l'IH au sens où en parlent ces auteurs. Comme ces derniers se préoccupent essentiellement de caractériser la problématique et les résultats de chacun de ces pôles, on ne peut tester cette hypothèse à cette étape. On ne peut le faire que si on remonte aux visions qui commandent ces trois choix distincts de méthode, afin de caractériser les approches correspondantes (on les note dans la suite AIR, AISo et AIH).

\subsection{L'approche en institutionnalisme rationnel (AIR)}

Au plan de la méthode, l'AIR est l'approche qui retient que « on doit partir des individus et on doit passer par des objets collectifs» (solution notée (2)). Les développements de la première partie nous permettent de caractériser sans problème la vision qui porte cette méthode.

La vision de l'AIR

Pour pouvoir partir des individus, il faut se donner une propriété qui est commune à tous les individus et sur laquelle on ne reviendra pas dans l'analyse. Cette propriété, ou logique d'action si on préfère, est la rationalité posée comme exogène. On voit donc cette rationalité, au moins instrumentale si ce n'est substantielle, à l'amont des comportements des individus et on ne voit pas d'objets collectifs à l'amont de cette rationalité. Comme on l'a déjà indiqué, cela signifie qu'on peut laisser entendre qu'il y en a, mais que ces objets collectifs ne sont pas ressaisis dans l'analyse que l'on développe (on ne se propose pas de comprendre ces objets comme des effets de composition - intentionnels ou in-intentionnels - des comportements des individus). Pour le dire autrement, la rationalité n'est pas prise dans une boucle causale systémique.

25 Economie et Institutions $-\mathrm{n}^{\circ} 4-1^{\mathrm{e}}$ semestre 2004 
Et si on doit passer par des objets collectifs, c'est que la rationalité ne suffit pas à déterminer les comportements. On voit aussi des règles à l'amont des comportements. Ces règles sont des contraintes «librement " acceptées par les individus rationnels. Elles ont donc été choisies par eux parmi un ensemble de solutions possibles. Il peut s'agir d'une norme macro-sociale ou d'un arrangement institutionnel, organisationnel ou contractuel méso-social. L'analyse théorique a pour principal objet d'expliquer quel est celle ou celui que les individus concernés vont choisir. La représentation schématique de l'explication des phénomènes sociaux dans cette approche est donc la suivante (voir figure 4.1).

Figure 4.1: L'approche en institutionnalisme rationnel (AIR)

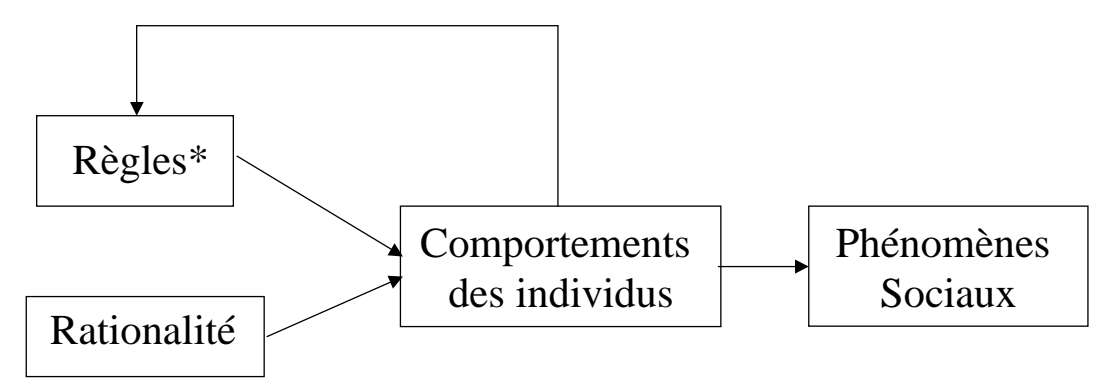
* Norme sociale particulière ou arrangement institutionnel, organisationnel ou
contractuel local (non compris l'environnement institutionnel exogène)

On est en présence d'une causalité circulaire. Mais il existe un moyen de conserver une démarche hypothético-déductive: procéder en deux temps [Charreaux, 1999]. Le type d'arrangement ressaisi est local, expression qui recouvre deux sens différents. Soit cet arrangement ne concerne qu'un nombre limité d'individus insérés dans un environnement institutionnel commun à un grand nombre et donné (lorsqu'il est explicité). Soit on se propose d'expliquer la formation d'une seule norme sociale commune à tous les individus de la société prise en compte et pouvant comprendre plusieurs règles élémentaires, en considérant le reste des règles sociales comme un environnement institutionnel donné21. Dans un cas

21 Cela est bien mis en évidence par [Cherkaoui, 2003] concernant la théorie de Coleman. Ce dernier se préoccupe essentiellement de produire une

Economie et Institutions $-\mathrm{n}^{\circ} 4-1^{\mathrm{e}}$ semestre 2004 
comme dans l'autre, l'analyse développée n'a pas pour objet d'expliquer la formation de cet environnement. L'AIR est une approche à portée locale. A environnement institutionnel donné, ce schéma contient un schéma explicatif de la formation de règles locales (voir figure 5.1).

Figure 5.1: La formation des règles dans l'approche en institutionnalisme rationnel (AIR)

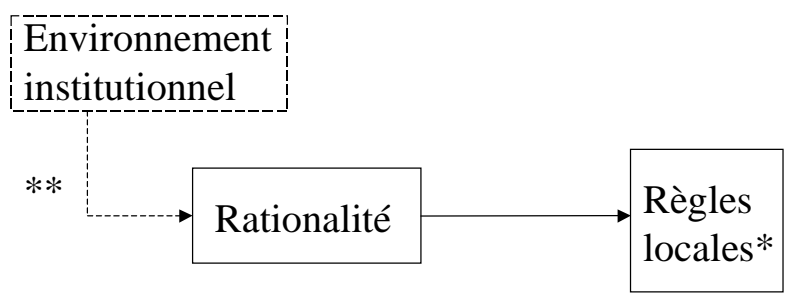

* Norme sociale particulière ou arrangement institutionnel, organisationnel ou contractuel local

** Eventuellement explicité

\section{Le statut du recouvrement de la PI par la PR : l'AIR n'est pas dans la PR}

Le propre de l'AIR au sein de la PI est donc de postuler, en fin de compte, une orientation téléologique des comportements (précisons que dans les figures ci-dessus, comme d'ailleurs dans les suivantes, la flèche qui part de la rationalité visualise une explication à orientation téléologique et celle qui part des règles, une explication à orientation causale). Cela justifie la dénomination retenue. Pour autant, l'AIR ne relève pas «en bloc » de la PR, puisque le choix d'une rationalité exogène y est méthodologique. Mais cela n'exclut pas que des chercheurs qui "ne laissent rien entendre" s'en réclament encore à juste titre en étendant son champ d'action, au delà de l'approche rationaliste standard (AR), en vue d'expliquer la formation des institutions. L'image qui nous paraît convenir est de dire que la PR se perd dans les sables dans cette tentative de dépassement de l'AR, parce qu'elle

théorie de la remontée du niveau micro (les actions individuelles) au niveau macro (l'effet sociétal ou résultats macro sociaux) [Steiner, 2003]. Cette analyse relève de l'AIR.

27 Economie et Institutions $-\mathrm{n}^{\circ} 4-1^{\mathrm{e}}$ semestre 2004 
n'attrape pas une autre approche, en l'occurrence l'AIR. Tel serait le statut du recouvrement entre la PR et la PI.

\subsection{L'approche en institutionnalisme sociologique (AISo)}

L'AISo est l'approche dont la norme méthodologique est $\mathrm{l}^{\prime} \mathrm{HM}$ faible : « on doit partir à la fois des individus et d'objets collectifs » (solution notée (3)). La vision qui commande cette norme méthodologique a deux caractéristiques.

La vision de l'AISo

Puisqu' on doit au moins partir des individus, on voit la rationalité à l'amont des comportements individuels; mais comme on ne peut seulement partir des individus, on voit cette rationalité comme endogène. La vision en question est donc holiste. Et comme on doit partir à la fois des individus et d'objets collectifs, il s'agit d'un holisme ensembliste.

Dans cette approche, ce n'est pas tant le fait que la rationalité s'exprime par des règles - c'est en suivant ces règles que l'individu se montre rationnel - qui est essentiel, puisque cela vaut déjà pour une rationalité procédurale de seconde nature (voir Coleman). Ce qui oppose cette approche à la précédente est que ces règles font partie de ce que la théorie se propose d'expliquer ou de comprendre. On se préoccupe de rendre compte de la formation sociale de la rationalité. Ces règles sont avant tout des conventions (ou croyances) d'ordre culturel. Leur institution est comprise comme le résultat de l'interaction synchronique des individus. En conséquence, ces règles sont déjà là avant les conduites menées rationnellement dans le cours $\mathrm{du}$ jeu social. Elles sont sociales. Telle est bien la proposition fondatrice de la sociologie pour Durkheim [1894]. C'est pour cette raison que je conserve l'expression d'approche institutionnaliste «sociologique» (AISo) pour qualifier cette seconde modalitée 22 . Le schéma explicatif des phénomènes sociaux est alors le suivant (voir figure 4.2).

22 On précise, de nouveau, que cette approche n'est pas l'approche des institutions retenue en sociologie, ni même celle de Durkheim. Par ailleurs, on ne peut retenir "interactionniste " [Grosseti, Filippi, 2004] dans la mesure où certaines analyses en AIR le sont.

Economie et Institutions $-n^{\circ} 4-1^{e}$ semestre 2004 


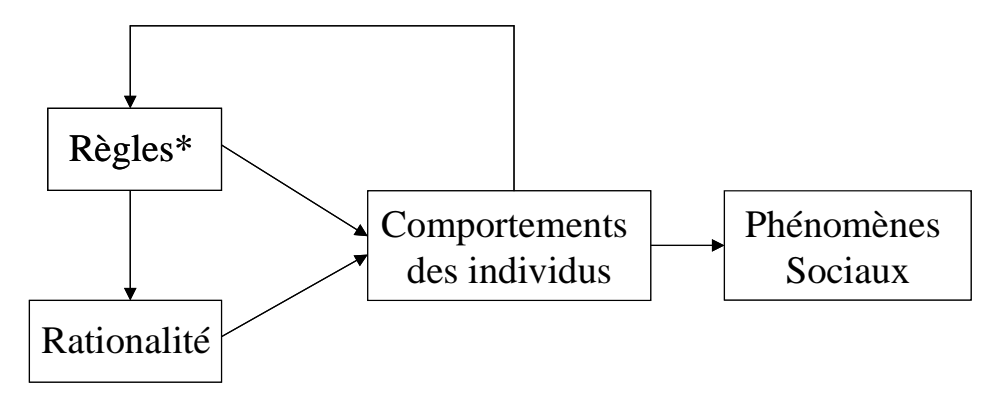

* Institutions, arrangements institutionnels, organisationnels ou contractuels

Comme pour l'AIR, cette façon d'expliquer les phénomènes sociaux contient une façon de comprendre la formation des règles (voir figure 5.2) ${ }^{23}$. Il ne s'agit plus, comme dans l'AIR, d'une explication produite de façon hypothético-déductive, mais d'une explication mettant en jeu une causalité circulaire, c'est-à-dire d'une compréhension. En tant qu'il est pensé en synchronie, le système en question ne peut s'analyser comme s'il comprenait une action et une rétroaction. Il y a parfaite symétrie. On doit faire appel à un principe de constitution pour penser une stabilisation de ce système selon une solution articulant un certain modèle de rationalité et des règles cohérentes avec ce modèle. Comme le retiennent les chercheurs qui s'inscrivent dans le programme de recherche de l'Economie des conventions ${ }^{24}$, un tel principe peut être qualifié de convention constitutive. Ce n'est pas une convention au sens courant du terme, c'est à dire une sorte de règle instituée. Elle est instituante. Elle préside à l'institution de conventions de comportement. C'est une grammaire de justification dont les individus se

23 Dans cette figure, les expressions utilisées - celle d' "holisme fonctionnel " pour qualifier le moment de l'action des règles sur la rationalité et celle d' "individualisme morphogénétique " pour qualifier le moment de l'action de la rationalité sur les règles - sont empruntées à Hervé Defalvard [1992 : 128], qui a proposé une première version de ce schéma en étudiant les travaux de l'économie des conventions. Dans cette version primitive, la rationalité et les comportements sont encore confondus. En effet, la causalité circulaire dont Defalvard fait état est celle entre une convention et le comportement des individus. Cela revient à ne pas distinguer les pôles " rationalité " et " comportement " et, par voie de conséquence, à confondre les deux circularités de la figure 4.2.

24 Voir notamment [Dupuy et ali, 1989], [Boltanski, Thevenot, 1991], [Eymard-Duvernay, 2002] et [Bessy, Favereau, 2003].

29 Economie et Institutions $-\mathrm{n}^{\circ} 4-1^{\mathrm{e}}$ semestre 2004 
servent pour exprimer les raisons pour lesquelles telle règle leur paraît socialement juste, en ce sens qu'elle sert le bien commun de la société. Elle est à la fois le fruit d'un accord - il n'y a pas d'institution de règles sans un accord sur une convention constitutive - et quelque chose qui leur est extérieur. Ni purement subjective, ni purement objective, elle est suprasubjective [Simmel, 1987: 47] : on est en présence d'une justification sociale commune (voir tableau 2 supra). On précise dans la troisième partie qu'il existe une pluralité de telles conventions constitutives de mondes communs justifiés.

\section{Figure 5.2: La formation des $r$ ègles dans l'approche en institutionnalisme sociologique( AISo)}

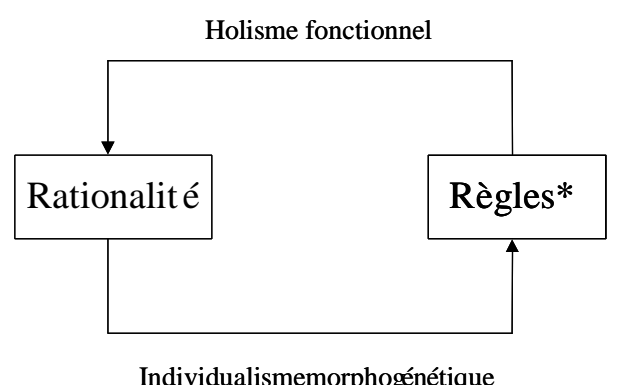

* Conventions, institutions, arrangements institutionnels, organtionnels ou contractuels

\section{L'AISo se positionne dans l'espace situé entre la PR et la PS}

L'AISo ne s'inscrit pas dans la PR, puisque la rationalité y est endogène. Cette approche est aussi extérieure à la PS, puisque sa vision n'est pas le holisme structural. Cette position intermédiaire se manifeste dans le fait que l'explication des phénomènes sociaux, comme celle des institutions (règles), relève d'un strict équilibre entre orientation causale et orientation téléologique. Tel est le sens de la symétrie dont on vient de parler.

\subsection{L'approche en institutionnalisme historique ( $\mathrm{AIH})$}

L'AIH est l'approche dont la norme méthodologique consiste à " partir d'objets collectifs et passer par les individus " (solution notée (4)). Comme pour les deux approches précédentes, il s'agit d'en préciser la vision 
et, en conséquence, la façon dont les phénomènes sociaux et la formation des règles sont expliqués.

\section{La vision de l'AIH}

La première caractéristique de la vision de l'AIH est d'être holistestructuraliste, puisqu' on doit partir uniquement d'objets collectifs. Comme on doit aussi passer par les individus, cela implique que ceux-ci soient des entités dotées d'une consistance. Il y a donc quelque chose à l'amont des comportements des individus qui doit être explicité, distinctement des objets collectifs qui sont par ailleurs à l'amont de ce quelque chose. Si on s'entend bien sur la définition que l'on a donnée de la rationalité (en général), cela ne pose aucun problème de dire que ce quelque chose est cette rationalité, même si ce terme n'est pas le plus souvent celui qui est employé par ceux dont les analyses relèvent du holisme structural, si ce n'est de cette approche ${ }^{25}$. Il va de soi que cette rationalité est vue comme endogène. Ce qui différencie nettement cette approche de la précédente est donc de donner une place centrale à l'action collective: il y a un processus de production de règles de comportements par l'action collective. Telle était déjà le point de vue de l'ancien institutionnalisme de la première moitié du vingtième siècle ${ }^{26}$. Et on doit ajouter que cette action collective est pensée en dynamique historique irréversible.

En résumé, la modification que l'on apporte à la vision de l'AISo est la suivante: on passe d'une rationalité endogène opérant en statique dans un monde apaisé à une rationalité endogène opérant en dynamique dans le cours d'un processus historique en permanence porteur d'un re

25 Ainsi, Giddens parle de "rationalisation conçue en tant que procès " [1987: 51], tandis que Bourdieu [1994] réserve le terme au cas de comportements réfléchis, de décisions précédées d'un raisonnement discursif

26 L'OI se composait d'une ancienne économie institutionnelle, dont Veblen et Commons furent les principaux représentants (ainsi que d'une ancienne sociologie institutionnelle identifiée par les travaux de Philip Selznick et de ses collègues en matière d'étude des organisations [DiMaggio, Powell, 1997]). La première s'est constituée en rupture par rapport à l'économie néoclassique. Cette rupture se traduit par les oppositions suivantes: économie " rationnelle " / économie "institutionnelle " au plan de la vision ; économie "statique" / économie "dynamique" au plan de la méthode; économie "spontanée " / économie "dirigée " (brain trust) au plan de la doctrine [Pirou, 1939 : 7]. On est en présence de premiers défrichements de l'action collective organisée pensée comme interaction entre structures et pratiques. On ne peut dire que l'on a déjà à l'œuvre une approche constituée. Reste que l'OI peut être classé a posteriori comme un institutionnalisme (à affinité) structuraliste [Bazzoli, 2001].

31 Economie et Institutions $-\mathrm{n}^{\circ} 4-1^{\mathrm{e}}$ semestre 2004 
surgissement du conflit social. En effet, cette vision fait une place essentielle au conflit social, c'est-à-dire à celui qui voit s'opposer des groupes d'individus (ou d'agents organisés) aux intérêts contradictoires - ce qui implique que les individus (ou les organisations agissantes) faisant partie d'un même groupe se sont reconnus solidaires les uns des autres via la formation d'acteurs collectifs. A la base, il y a toujours, selon les termes de Commons, un "conflit de prétention" (content of wills) en matière de définition ou de transfert de droits de propriété dans toute interaction entre deux ou plusieurs personnes qui sont dépendantes les unes des autres, l'une ne pouvant parvenir à ses fins sans l'autre. Ce conflit devient social lorsque, d'un côté au moins, des personnes se trouvent dans la même situation de dépendance et agissent de concert pour défendre leur intérêt commun. La situation renvoie à la structure et le règlement du conflit, à une forme institutionnelle. Ce règlement du conflit social ne relève pas le plus souvent d'une justification commune (AISo), mais d'une justification en droit de nature procédurale. Cette dernière est strictement collective (voir tableau 2 supra). Elle se présente comme un compromis entre des points de vue diversement justifiés en termes de justification commune. Ce compromis traduit un certain état du rapport des forces en présence ${ }^{27}$. Ainsi, l'ordre instauré, qui ne perdure que si les pratiques des individus l'actualisent, n'a pas supprimé le conflit. Il est à même de resurgir à tout moment. Il est le moteur du changement.

A s'en tenir à la vision de l'AISo, le conflit a disparu ; s'il est présent lorsqu'il s'agit de s'entendre sur une convention constitutive, il a été dissout dans la solution de coordination retenue pour construire un monde; ce dernier est apaisé ; le conflit ne peut réapparaître sur le devant de la scène. On ne peut en comprendre la résurgence qu'à deux conditions : 1/ adopter une conception strictement dynamique de la rationalité - elle s'exprime en termes «d'évolution satisfaisante » [Billaudot, 2001 : 193] ; 2/ introduire un effet en retour, par nature diachronique, des conditions de déroulement des phénomènes sociaux sur la rationalité [Goldmann, 1977]. La rationalité est donc comprise comme un effet du processus de structuration de l'individu, processus dont la cohérence est toujours partielle. Cela laisse place à l'ouverture sur du nouveau imprédictible; autrement dit, à l'invention et à la création ${ }^{28}$. Cette ouverture potentielle du processus de structuration de l'individu est tout à la fois le reflet et la condition de l'ouverture du processus de structuration sociale.

27 D'un point de vue subjectif, chacun est en droit de considérer qu'un comportement légal n'est pas juste au regard de tel ou telle grammaire de justification à laquelle il se réfère. L'ordre "de droit " sera alors considéré comme un désordre établi (E. Mounier).

28 "Quand vous êtes complètement ouvert, l'intérêt de chacun devient votre propre intérêt. Alors règne une liberté absolue " [Srinivasan, 1984 : 55].

Economie et Institutions $-\mathrm{n}^{\circ} 4-1^{\mathrm{e}}$ semestre 2004 
En $\mathrm{AIH}, \mathrm{l}$ 'analyse est donc strictement dynamique: tout état à un moment donné n'est qu'une photographie d'un processus en train de se faire. La rationalité commande d'actualiser les règles si l'évolution constatée par l'individu est conforme à la représentation associée à la justification subjective du suivi de la règle; c'est-à-dire, si elle correspond à l'évolution attendue constitutive de cette représentation ${ }^{29}$. Sinon, une tension se manifeste. La résurgence du conflit social naît de tensions sociales. L'analyse ne peut prédire si cette tension va déboucher sur une grande crise ou se résorber. La résorption passe par la mise en place de règles commandées par le second niveau d'apprentissage de Argyris et Schön [1978] ; c'est-à-dire, par la construction de règles qui permettent de se sortir de difficultés habituelles. On pourra dire après coup que ce fût une petite crise. Une grande crise se constate lorsque ces règles ne répondent plus à leur justification: elle produit un changement de tout ou partie des formes institutionnelles ${ }^{30}$.

On voit donc le système de formes institutionnelles transitoirement ou durablement stabilisées, par lequel se manifeste l'existence de toute société ou de tout ensemble social concret, comme le point de sortie temporaire ou le point d'aboutissement d'une grande crise d'un ancien système. On le regarde comme le résultat de la mutation de ce dernier, en raison de son entrée en crise. C'est la cohérence des diverses formes les unes avec les autres qui fait la sortie de crise. On ne peut en penser la formation séparément (à la différence de l'AIR qui est une approche à portée locale). $C^{\prime}$ est un processus de transformation. En cela, cette vision est structuraliste ; elle relève du holisme structural. La rationalité devient une rationalité située, en ce sens précis que les conventions de comportement et les modes d'interprétation des règles de droit qu'elle assure sont relatifs à la place occupée dans la structure. Cette approche est à portée globale. On se préoccupe de rendre compte du système des formes institutionnelles en place, c'est-à-dire de la complémentarité et de la hiérarchie de ces formes.

En conséquence de cette révision, le schéma explicatif des phénomènes sociaux propre à l'AIH se présente comme une version dynamisée et historicisée de celui de l'AISo (voir figure 4.3). Ce schéma contient une compréhension de la formation des règles (voir figure 5.3).

29 Cette représentation de l'effet attendu d'une action est toujours partielle et subjective. Elle n'implique aucunement que chaque individu dispose dans son capital de connaissances ou son savoir d'un modèle du déroulement de la vie sociale dans son ensemble.

30 Cette logique d'analyse est celle, en économie, vers laquelle tend l'Ecole de la régulation (voir notamment [Boyer, 2003a, 2003b], [Chanteau, 2003], [Chavance, 2001] et [Servais, 2000]).

33 Economie et Institutions $-\mathrm{n}^{\circ} 4-1^{\mathrm{e}}$ semestre 2004 
Figure 4.3 : L'approche en institutionnalisme historique (AIH)

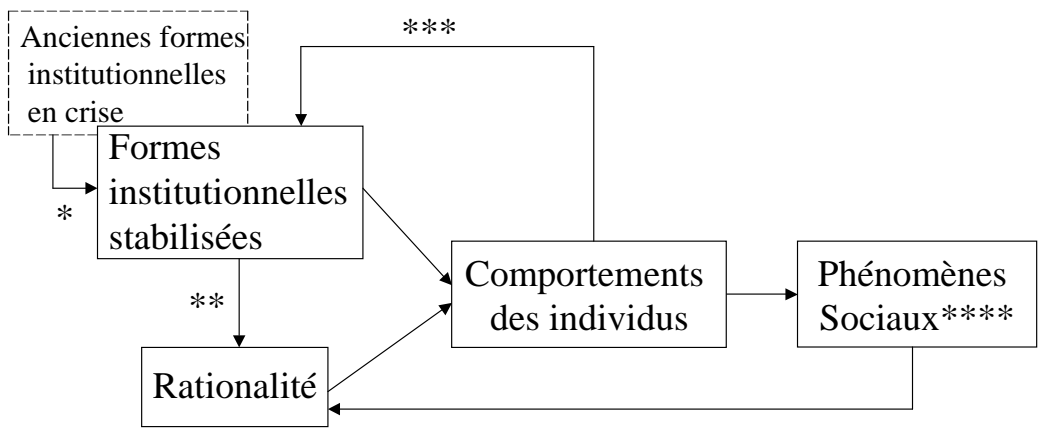

* Processus de sortie de crise : genèse des formes institutionnelles

** Formation de la rationalité située (dans l'histoire)

*** Actualisation des règles en vigueur par des pratiques conformes à ces règles

**** Phénomènes en régime

Figure 5. 3 : La formation des règles dans l'approche en institutionnalisme historique (AIH)

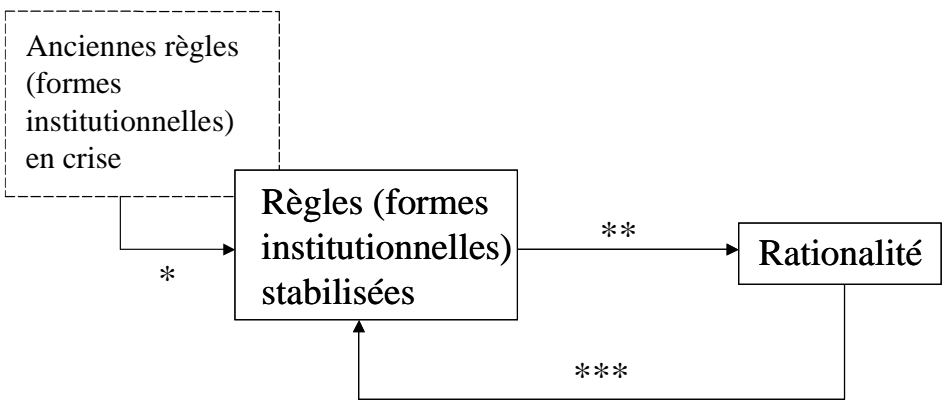

* Processus de sortie de crise : genèse des formes institutionnelles

** Formation de la rationalité située (dans l'histoire)

*** Justification a posteriori 
L'AIH est une approche institutionnaliste qui est en même temps structuraliste. Elle occupe l'espace de recouvrement de la PI par la PS. Cette double appartenance est rendue manifeste par le fait que l'explication donnée tant des phénomènes sociaux que de la formation des règles est, de façon dominante, à orientation causale. L'orientation téléologique est présente, mais elle est englobée. On est en présence d'un déterminisme complexe qui interdit de pouvoir prédire l'avenir.

2.6. Conclusion : trois approches distinctes

On a ainsi construit trois approches tout à fait distinctes les unes des autres (voir tableau 5).

Tableau $5: \underline{\text { Les trois approches institutionnalistes }}$

\begin{tabular}{|l|l|l|}
\hline Type & Vision & Méthode* \\
\hline AIR & $\begin{array}{l}\text { Individualiste } \\
\text { Rationalité (naturelle ou sociale) } \\
\text { exogène }\end{array}$ & $\begin{array}{l}(2)^{*} \text { « On doit partir des individus } \\
\text { et passer par des objets collectifs » } \\
\text { (partie de l'IM simple ) }\end{array}$ \\
\hline AISo & $\begin{array}{l}\text { Holiste ensembliste } \\
\text { Rationalité (sociale) endogène opérant } \\
\text { en état }\end{array}$ & $\begin{array}{l}(3)^{*} \text { « On doit partir à la fois des } \\
\text { individus et d'objets collectifs » } \\
\text { (partie de l'IM complexe : } \\
\text { HM faible) }\end{array}$ \\
\hline AIH & $\begin{array}{l}\text { Holiste structuraliste } \\
\text { Rationalité (sociale) endogène opérant } \\
\text { en dynamique }\end{array}$ & $\begin{array}{l}(4)^{*} \text { «On doit partir d'objets } \\
\text { collectifs et passer par les } \\
\text { individus » (partie de l'HM normal) }\end{array}$ \\
\hline
\end{tabular}

* voir tableau annexé à la figure 3.

Cette construction ne doit rien dans son élaboration aux pratiques effectives de recherche, notamment à celles qui ont été prises en compte par Hall et Taylor, puis par Théret. On ne doit pas toutefois être victime de l'illusion que ce constat peut véhiculer, celle qui consisterait à croire que cette construction serait en quelque sorte la vérité sur les approches possibles, sans lien avec l'histoire des pratiques effectives ; autrement dit, en laissant entendre qu'on aurait pu construire cela dans le passé et que l'on n'aura aucun changement à y apporter dans l'avenir. En effet, cette construction est contingente, dans la mesure où elle a pris comme point de départ l'état actuel du débat individualisme méthodologique/holisme méthodologique.

35 Economie et Institutions $-\mathrm{n}^{\circ} 4-1^{\mathrm{e}}$ semestre 2004 
Cela a toutefois un sens de tester le bien fondé de cette construction au regard des pratiques effectives de recherche. Est-on en présence d'une cohérence entre cette construction normative selon laquelle il y a trois approches institutionnalistes possibles (et seulement trois) et le constat que les travaux effectifs peuvent être classés en trois groupes distincts? La cohérence est acquise au plan sémantique, mais elle ne se constate que très partiellement sur le fond. On ne peut en comprendre la raison qu'après avoir vu comment ces trois approches s'articulent.

\section{La déclinaison de la problématique institutionnaliste en AIR, AISo et AIH : trois niveaux successifs de prise en compte de la complexité de la vie sociale}

L'analyse des trois approches institutionnalistes a conforté l'hypothèse d'une réelle consistance de la PI. Cette dernière se présente comme une structure dont l'AIR, l'AISo et l'AIH sont des modèles. On a explicité certaines caractéristiques des transformations par lesquelles on passe de l'un à l'autre de ces modèles. Il nous reste à traiter de la logique de ce processus de transformation.

La proposition qui est maintenant défendue est la suivante : la PI se décline au pluriel parce qu'on a divers degrés possibles de complexité dans sa mise en œuvre; ces degrés correspondent à des étapes, de la plus simple à la plus complète, pour appréhender la complexité de la vie sociale; cette dernière tient essentiellement à la différence et au changement. C'est par là qu'il faut commencer.

3.1 La complexité de la vie sociale: différence et changement. Une hypothèse sur son traitement

A l'observation, on peut faire deux constats : les comportements des individus placés dans une même situation sont différents d'un individu à l'autre à un moment donné du temps et ils changent dans le temps. D'un point de vue objectif dans le temps chronologique (historique), les différences s'observent en synchronie et les changements, en diachronie. Doit-on prendre en compte ces différences et ces changements pour comprendre les phénomènes sociaux ? On ne peut formuler une réponse à cette question que si on s'entend sur ce qu'est une différence, et sur les raisons pour lesquelles le changement n'est pas assimilable à une différence. 


\section{La différence suppose une vision holiste de la vie sociale}

On ne peut parler de comportements différents qu'à propos d'une même situation et l'on ne peut parler théoriquement d'une même situation que si on se réfère à une structure ou au moins à un tout qui est plus que les éléments qui le constituent. Cette même situation est une certaine place dans cette structure ou une inscription dans ce tout. Si on n'adopte pas une vision holiste (structuraliste ou ensembliste) de la vie sociale, on ne peut pas comprendre ce qu'est une différence. On peut seulement introduire a priori de façon exogène des différences à partir d'une indifférenciation première des individus, comme on l'a vu à propos de la fonction de satisfaction en rationalité instrumentale. Précisons qu'on ne s'intéresse qu'aux différences sociales abstraites [Caillé, 1986] ou d'ordre systémique [Giddens, 1987], non aux différences proprement personnelles.

\section{Le changement n'est pas assimilable à la différence}

Un changement de comportement est relatif à une même place dans une structure. Un changement signifie que l'on a l'apparition dans l'histoire d'un nouveau comportement à cette place. Sa nouveauté réside dans le fait qu'il n'est pas réductible à un type de comportement passé. Il n'est pas compréhensible avec les moyens qu'on s'est donné pour comprendre les différences que l'on a pu constater à un moment quelconque du passé.

Cela n'interdit pas d'adopter une démarche dans laquelle on fait comme si le changement était soluble dans la différence. La réduction en question est la suivante. Ayant constaté que du temps $t$ au temps $t+1$ le comportement $\mathrm{d}^{\prime}$ individus a changé en passant du type $\mathrm{A}$ au type $\mathrm{A}^{\prime}$, on fait comme si $A^{\prime}$ est un comportement $B$ théoriquement observable en $t$; on fait donc comme s'il n'y a jamais rien de vraiment nouveau qui puisse voir le jour dans le temps historique; les seuls changements sont de l'ordre des proportions entre des éléments dont la liste est connue de façon a temporelle. Il n'en reste pas moins que la nouveauté échappe à cette analyse réductrice.

\section{Une hypothèse sur la déclinaison de la problématique institutionnaliste}

Ce qui précède nous conduit à dire qu'il y a, en principe, trois degrés dans la prise en compte de la complexité de la vie sociale. Le degré zéro est celui qui ne se préoccupe de ressaisir ni les différences ni les changements. Au degré suivant (second niveau), on ressaisit les différences en réduisant le changement à de la différence. Au degré supérieur (troisième niveau), on ressaisit les deux sans aucune réduction. L'hypothèse faite est que la

37 Economie et Institutions $-\mathrm{n}^{\circ} 4-1^{\mathrm{e}}$ semestre 2004 
problématique institutionnaliste se décline à ces trois niveaux (voir tableau $6)^{31}$.

Tableau 6 : La déclinaison de la PI

\begin{tabular}{|l|l|}
\hline Approche & Degré de prise en compte de la complexité de la vie sociale \\
\hline AIR & On ne ressaisit ni les différences, ni les changements \\
\hline AISo & On ressaisit les différences, en réduisant le changement à de la différence \\
\hline AIH & On ressaisit les différences et les changements \\
\hline
\end{tabular}

\subsection{L'approche en institutionnalisme rationnel (AIR) : on ne ressaisit ni les différences ni les changements}

La forme la plus simple de mise en œuvre de la problématique institutionnaliste est celle qui ne se propose de ressaisir ni les différences ni les changements. Il s'agit de l'AIR. Puisque les règles sociales sont globalement les mêmes pour tous les individus, les différences de comportement ne peuvent provenir que de la rationalité. Et, lorsqu'on retient que cette dernière est exogène, on ne peut que les ignorer ou les prendre de facto comme exogènes. C'est la façon la plus simple de lever l'indétermination dont on a fait état dans la présentation de la PI.

Cette approche n'est pas muette sur la question du changement, mais ce dernier ne peut venir que d'un changement de l'environnement institutionnel ou d'un changement de la fonction de satisfaction de certains agents lorsqu'on ne rattache pas explicitement la rationalité à l'environnement institutionnel. En tout état de cause, ce changement est exogène. La solution simple est alors de raisonner en temps réversible; on ramène la dynamique à de la statique comparative; il y a alors réduction du changement diachronique à de la différence synchronique. Mais on peut aussi retenir que ce changement exogène est l'effet du hasard dans un temps irréversible; on est alors en présence d'une modalité d'institutionnalisme « évolutionniste » dans laquelle c'est le hasard qui est créateur [Lestienne, 1993]. Mais, même dans ce cas, ce n'est pas en fin de compte une solution qui nous fait sortir de l'AIR.

31 Faut-il faire une place à une quatrième stratégie de recherche, celle qui se proposerait de ressaisir le changement en faisant comme si la différence était soluble dans le changement? On peut monter, sans difficulté particulière, qu'il ne peut s'agir que d'une forme frustre d'AIH.

Economie et Institutions $-\mathrm{n}^{\circ} 4-1^{\mathrm{e}}$ semestre 2004 
Ainsi envisagée, l'AIR ne se présente donc pas comme une approche fermée réduite à un pôle - ce qu' elle serait par exemple si elle se limitait au cas particulier où l'hypothèse faite est celle d'une rationalité substantielle en temps réversible. C'est une approche ouverte, en ce sens qu'elle délimite un champ de programmes de recherche (ou de théories) plus précises se distinguant les uns des autres par la nature (naturelle/sociale) et la forme de la rationalité postulée, ainsi que par le mode de traitement du temps.

\subsection{L'approche en institutionnalisme sociologique (AISo) : on réduit le changement à de la différence}

La seconde modalité de mise en œuvre de la PI par ordre croissant de complexité est celle qui ressaisit les différences, tout en conservant cette réduction du changement à la différence dont on vient de faire état pour l'AIR. La montée en complexité consiste à passer d'une rationalité exogène à une rationalité endogène ${ }^{32}$. Cette solution de levée de l'indétermination première de la vision de la PI suffit à atteindre l'objectif visé.

En effet, le propre de la proposition constitutive de l'AISo (voir figure 5.2 supra) est de n'être prédictive d'aucune façon de tel ou tel système « règles/rationalité » (ou encore «justice/intérêt»). Si on est capable de décliner un certain nombre de principes de constitution du système en question, c'est-à-dire un certain nombre de conventions constitutives, on produit de la différence. En effet, on associe à chaque convention constitutive un ensemble de comportements tels que d'un ensemble à l'autre le comportement des individus sera différent. Chacun des systèmes ainsi construits de façon moniste peut être qualifié de monde, de cité, de système social et culturel, de modèle social ou culturel, peu importe ${ }^{33}$. Cela s'accorde à la vision de l'AISo qui relève seulement d'un holisme ensembliste, vision

32 Cela n'implique pas, pour autant, que tout ce qui a été fait en AIR devient caduc. Au contraire, on peut se servir des analyses qui ont été développées dans ce cadre, à condition de ne pas adopter leurs conclusions. Cela implique toutefois que le passage d'un étage (AIR) à l'autre (AISo) soit possible c'est-à-dire que l'on ait en bas (premier niveau) comme en haut (second niveau) le même type de rationalité (voir figure 6 infra).

$33 \mathrm{Au}$ sein de l'Economie des conventions (EC) par exemple, il est couramment fait état, à la suite de Boltanski et Thévenot [1991], de six principes supérieurs représentant les six conceptions du juste faisant l'objet d'un consensus dans une société démocratique en se référant à de grands penseurs: les principes marchand, industriel, civique, de l'opinion, domestique, inspiré. Mais la liste de ces principes n'est pas fermée a priori, puisque dans un ouvrage ultérieur [Boltanski, Chiapello, 1999] il est fait état d'une nouvelle cité, la " cité par projets". Pour sa part, Robert Salais [1998] en limite le nombre à quatre en s'appuyant sur la théorie de la justice de Rawls.

39 Economie et Institutions $-\mathrm{n}^{\circ} 4-1^{\mathrm{e}}$ semestre 2004 
selon laquelle il y a seulement une commune appartenance de chaque individu à l'ensemble sur la base d'une identité individuelle partagée. Chaque monde est alors une certaine façon de sceller cette commune appartenance. Tous les membres de ce monde se réfèrent à la même grammaire pour justifier les règles du jeu social. Il n'y a de différences qu'entre mondes, la même rationalité propre à un monde opérant dans toutes les relations. On comprend alors toute société concrète en combinant plusieurs mondes en certaines proportions et l'on est en présence d'une épreuve de coexistence entre ces mondes. On ne peut comprendre cette coexistence, qui tient à la présence de règles de droit irréductibles à une cité particulière, dans le cadre de l'AISo.

Ce mode d'analyse n'interdit pas de dire quelque chose à propos des changements des comportements dans le temps. Seulement, ils sont réduits à des différences. Le propos tenu est, en effet, que ces changements s'expliquent par un changement de proportions entre les modèles qui coexistent, quitte à inventer alors un nouveau modèle. Il n'en reste pas moins que ce dernier ne doit rien à l'histoire...non pas dans la réalité, mais dans l'analyse qui le produit! On n'explique pas la création de ce nouveau modèle au sein d'un processus historique. Ce serait quitter le plan de l'AISo, en montant au niveau de $\mathrm{l}^{\prime} \mathrm{AIH}^{34}$.

Comme on l'a déjà vu pour l'AIR, c'est aussi d'un plan dont il s'agit : l'AISo délimite un champ au sein duquel une grande diversité de spécifications peut exister. Cela concerne principalement le moment de l'analyse relatif à la formation sociale de la rationalité. Certaines problématiques sont tournées vers l'AIR, d'autres vers l'AIH ${ }^{35}$. Tant qu'elles restent inscrites dans l'AISo, toutes ces problématiques ont en commun une même stratégie de recherche dite holindividualiste au sens de Defalvard [1992, 2000], c'est-à-dire une stratégie qui vise à coupler un moment holiste (orientation causale) et un moment individualiste (orientation téléologique) sans préséance de l'un sur l'autre. On n'a pas à se préoccuper de savoir de quoi on doit partir, puisque le mode hypothético-déductif n'est mis en œuvre que pour l'analyse de chacun des deux moments en question. La méthode de l'AISo est donc bien, à l'image du holisme ensembliste dont sa vision relève, une démarche intermédiaire entre l'IM simple et l'HM normal. Le passage à l'AIH implique une appropriation de cette stratégie de

34 Rappelons à ce titre que l'AISo et l'EC ne sont pas de même nature. L'EC est un programme de recherche qui suit une trajectoire l'ayant conduit à se positionner en AISo et à s'en éloigner vers l'AIH dès lors qu'il se préoccupe de ressaisir les règles de droit et qu'il traite de la naissance d'une nouvelle cité.

35 Ceci est reconnu au sein de l'économie des conventions, si ce n'est au départ dans [Dupuy et alii, 1989], du moins au-delà dans [Batifoulier, 2001], [Eymard-Duvernay, 2002] et [Ughetto, 2003].

Economie et Institutions $-n^{\circ} 4-1^{e}$ semestre 2004 
recherche en un sens qui lève la tension inhérente à cette position intermédiaire.

\subsection{L'approche en institutionnalisme historique (AIH) : on ressaisit différences et changements}

L'objet essentiel de l'AIH est de ressaisir les changements des comportements individuels dans l'histoire. En conformité avec la PI, c'est à cette condition que l'on doit être à même de comprendre à la fois le changement historique des institutions et celui des phénomènes sociaux. L'hypothèse est que le passage de l'AISo à l'AIH a cet enjeu. Tel est bien le cas puisqu' on a analysé la vision de l'AIH comme une version dynamisée et historicisée de celle de l'AISo. Les schémas explicatifs et la démarche d'analyse s'en trouvent profondément modifiés. Le niveau de complexité auquel on doit monter n'implique pas toutefois de faire table rase de tout ce qui a été fait en AIR et en AISo.

En couplant en synchronie le moment de l'action de la rationalité sur les règles via les comportements des individus (liaison déjà analysée dans $l^{\prime}$ AIR) et le moment de l'action des règles sur la rationalité (moment propre à l'AISo), cette seconde étape dans la mise en œuvre de la PI rend compte d'une cohérence interne à un système. D'un point de vue historique, il s'agit d'une forme structurelle qui a une existence non éphémère. La construction théorique réalisée nous dit seulement que c'est une forme qui peut durer. Elle ne nous permet pas de comprendre le processus de son avènement (ou de sa genèse, si on préfère), ni d'ailleurs celui de sa transformation incrémentale - la même forme perdure - ou de sa transformation radicale une autre forme prend sa place. La première implication du changement de niveau à réaliser est d'introduire le concept de forme historique, s'appliquant aussi bien à une structure qu'à l'une ou l'autre des institutions comprises dans cette structure. Doit-on retenir qu'une forme structurelle historique est seulement un modèle de la structure dont le caractère spécifique est d'être nouveau dans l'histoire ? Ce serait négliger le fait qu'à un moment donné du temps, l'analyse de toute société ou ensemble social concret met en jeu une coexistence de plusieurs modèles. Une forme structurelle historique concrète est donc une nouvelle combinaison de modèles de la même structure, combinaison qui comprend elle-même un ou plusieurs nouveaux modèles. Quand au terme de forme institutionnelle, il peut être utilisé en deux sens différents : il désigne soit l'institutionnel de cette forme structurelle historique concrète, soit seulement la forme d'une institution dans l'un des modèles de la structure ${ }^{36}$. En tout état de cause,

36 Ces deux sens se confondent lorsqu'on s'en tient à la forme frustre d'AIH qui laisse de côté la différence, en ne retenant à chaque moment que le

41 Economie et Institutions $-n^{\circ} 4-1^{e}$ semestre 2004 
cette forme est rendue manifeste par des règles courantes actualisées par des pratiques.

On a vu ce que mettait en jeu le processus d'apparition d'une nouvelle forme institutionnelle. Dans le second schéma qui visualise ce processus (voir figure 5.3), l'action de la rationalité sur les règles (notée ***) est conçue comme un effet en retour, une rétroaction qui n'explique pas la genèse des règles ; cet effet est ambivalent puisqu'il les stabilise en régime et les déstabilise en crise. Ce lien a le statut d'une justification a posteriori : l'explication de l'AIR devient la justification savante des raisons pour lesquelles le suivi des règles en vigueur répond à l'intérêt bien compris de l'acteur individuel dans la situation où il se trouve ${ }^{37}$. On se sert donc de ce qui est fait en AIR et en AISo pour construire en AIH.

modèle dominant. On peut voir cette forme comme une étape dans la mise en œuvre de l'AIH, qui "saute " par-dessus l'AISo (flèche notée a dans la figure 6 infra) et par laquelle on peut passer à la condition de ne pas y rester (voir la première génération de travaux de l'école de la régulation). Précisons de plus que le second sens s'accorde à la proposition selon laquelle $1 /$ une structure est "formée par la convertibilité réciproque des règles et des ressources engagées dans la reproduction sociale "[Giddens, 1987 : 244] et 2/ l'institutionnel de la structure - se déclinant en une ou plusieurs institutions - en est le côté règles [Billaudot, 2002, 2004b]. C'est une façon particulière de donner un sens précis à la proposition selon laquelle l'institutionnel est une médiation entre la structure et les comportements [Theret, 2003].

37 "A posteriori " veut dire que ce discours n'aurait pu être tenu dans le cours du débat portant sur cette éventuelle institution que si l'environnement institutionnel futur était déjà là. Tel est, de mon point de vue, le canal essentiel de la "réflexivité de la science sociale " dont parle à juste titre Coleman [Foundations : 610], cité par Steiner [2003 : 218]. La justification savante en question n'est pas une mystification (aliénante au sens de Marx). Elle est fondée, toutes choses égales par ailleurs notamment, l'état du rapport des forces qui a présidé à la genèse des règles. On est en crise lorsque la justification est mise en échec - le suivi des règles s'accompagne d'un résultat qui n'est pas conforme à la prédiction contenue dans cette justification - ; la rationalité l'invite alors à exercer sa liberté (action déstabilisatrice-déstructurante) ou à se résigner. Ce choix n'est pas déterminé d'avance : la première branche de l'alternative comprend le couple "défection (exit)/prise de parole (voice) " de Hirschman [1986] sans s'y réduire, tandis que la seconde correspond, en première analyse, à la troisième solution de ce dernier (loyalty). C'est à ce moment qu'il convient de ressaisir le niveau de la socialité primaire, les différences personnelles étant en jeu dans ce choix. Ces différences relèvent-t-elles du hasard ou doit-on remonter dans la compréhension des causes ? Ce débat est ouvert au sein de l'Ecole de la régulation [Amable, Palombarini, 2003].

Economie et Institutions $-\mathrm{n}^{\circ} 4-1^{\mathrm{e}}$ semestre 2004 
En résumé, on peut dire que la vision de l'AIH est holindividualiste en un sens qui diffère de celui évoqué précédemment à propos de la vision de l'AISo dans laquelle on a une symétrie entre les deux composantes [Billaudot, 1996]. En AIH, la composante holiste est première (voir l'action notée * des figures 4.3 et 5.3), parce que les nouvelles règles ne se forment pas dans un vide institutionnel, mais dans un contexte de crise spécifique. La composante individualiste (orientation téléologique) vient ensuite, en nous invitant à passer par les individus pour comprendre à la fois l'actualisation des règles et les phénomènes sociaux. La méthode générale, qui s'accorde à cette vision, est un mode de compréhension post factum dont les trois temps sont observer, caractériser et comprendre ${ }^{38}$.

\section{Conclusion}

Les analyses prises en compte au point de départ de cet article sont confirmées sur un point essentiel. Si le renouveau de l'institutionnalisme est multiple, il y a trois façons tout à fait distinctes de mener une analyse qui soit apte à ressaisir les institutions en expliquant a priori ou en comprenant post factum leur formation. Ces analyses ont été complétées et amendées.

Le complément porte sur le fond commun de cette triade: la problématique institutionnaliste. Cette dernière se distingue à la fois de la problématique rationaliste et de la problématique structuraliste. Elle en est une combinaison appropriée: considérer que les comportements des individus modernes sont déterminés à la fois par des règles (relevant du registre de la justice au niveau macro) et par la rationalité (exprimant l'intérêt propre de l'individu au niveau micro). L'institutionnalisme rationnel, l'institutionnalisme sociologique et l'institutionnalisme historique se présentent alors comme trois façons distinctes de concevoir cet «à la fois ", selon que l'on introduit ou non une relation de cause à effet des règles sur la rationalité et selon le statut de cette relation lorsqu'elle est introduite. Comme solution intermédiaire, la PI disqualifie les deux problématiques traditionnelles et tend à s'imposer comme la seule problématique en science sociale. C'est à l'intérieur de cette nouvelle problématique que se réinvestit le débat rationalisme/structuralisme (voir l'opposition entre l'AIR et l'AIH).

L'amendement est de ne pas voir ces trois modalités comme trois pôles qui auraient à chaque fois deux à deux quelque chose en commun qui les opposerait au troisième - ce qui est toujours le cas lorsqu'on construit une cartographie à partir d'axes de différentiation combinés dans un même

38 Voir [Billaudot, 2004a], ainsi que [Sapir, 2003].

43 Economie et Institutions $-n^{\circ} 4-1^{\text {e }}$ semestre 2004 
plan ${ }^{39}$. Il s'agit de trois plans (voir figure 6). Cette représentation par plans successifs visualise le fait que ces trois approches ne sont pas de même niveau de complexité. L'approche en institutionnalisme rationnel ne se préoccupe pas d'expliquer les différences entre les comportements des individus et le changement dans le temps de ces comportements, en retenant que la rationalité est exogène. En considérant qu'elle est endogène, c'est-àdire qu'elle s'exprime par des règles (conventions) qui dépendent du monde dans lequel s'inscrit l'individu et qui doivent être ressaisies par l'analyse, l'approche en institutionnalisme sociologique prend en compte la différence en ignorant encore le changement. En ajoutant que les règles en vigueur sont un produit historique, l'approche en institutionnalisme historique a l'ambition de rendre compte à la fois de la différence et du changement.

\section{Figure 6: Les trois plans de la problématique institutionnaliste}

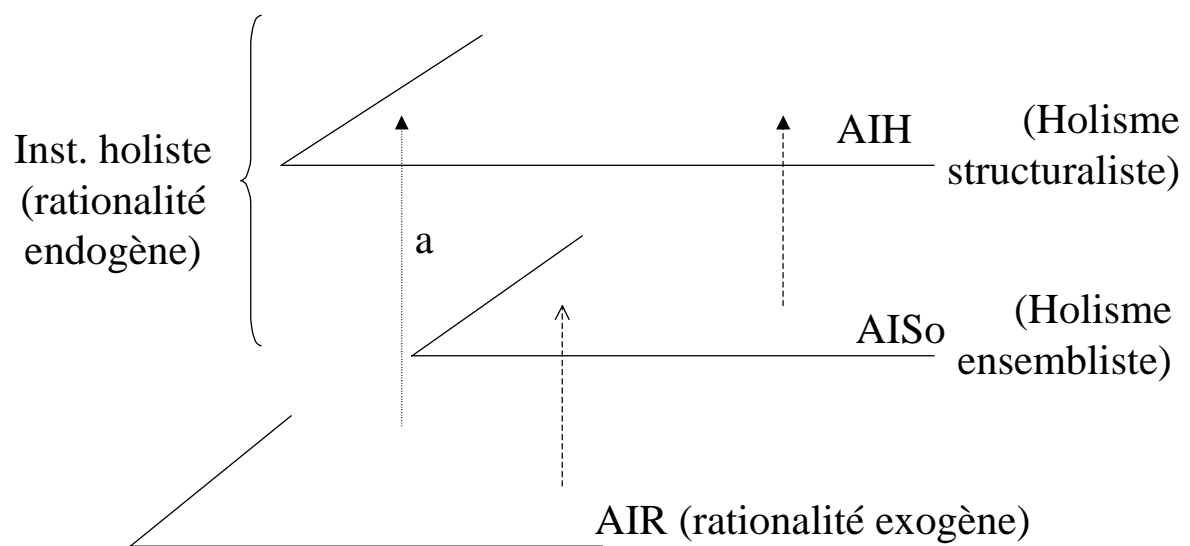

Lorsqu'on les envisage comme des étapes méthodologiquement justifiées par le souci de ne pas embrasser d'un coup la réalité sociale dans toute sa complexité, ces trois approches sont complémentaires. Elles le sont dans un sens tout à fait particulier. Il ne s'agit pas de les combiner en

39 Ainsi, Theret retient que l'IR met en jeu une "perspective calculatrice ", l'ISo une "perspective culturelle " tandis que l'IH " conjuguerait ces deux perspectives " (voir supra note 4). Or, s'il s'avère que la norme AIR avec une rationalité exogène et la norme AISo avec une rationalité endogène mettent bien en jeu les deux perspectives opposées en question, on ne parvient en aucune façon à la même concordance pour la norme $\mathrm{AIH}$, puisque dans cette troisième approche la rationalité est tout aussi endogène que dans la seconde. 
prenant dans chacune ce qu'il y aurait de bon. Ce sens précis est le suivant: on se sert de l'analyse réalisée en AIR pour construire en AISo et on se sert ensuite de l'analyse développée en propre au niveau de l'AISo pour construire en $\mathrm{AIH}$. Au premier de ces deux passages, il s'agit essentiellement de l'analyse que Coleman considère comme le lieu crucial de la théorie sociale contemporaine, celle qui montre comment les comportements individuels produisent telle ou telle règle (ou arrangement de règles) à partir d'une rationalité déduite d'un environnement institutionnel exogène. Au second passage, il s'agit essentiellement de l'analyse qui se propose de comprendre cette déduction en considérant que cet environnement est globalement endogène aux comportements individuels et en retenant que les règles ne s'exercent pas sans qu'elles ne soient préalablement interprétées ; autrement dit, on se sert de l'analyse qui couple structuration sociale et structuration de l'individu et qui fait une place dans toute " décision » d'un acteur de suivre une règle, en faisant appel à sa conscience pratique ou à sa conscience discursive, à la justification pour lui et pour les autres de l'intérêt de se comporter de cette façon. En fin de compte, seule l'AIH permet $\mathrm{d}$ 'articuler l'analyse locale de telle ou telle institution et l'analyse globale du système institutionnel en place à l'échelle d'un territoire ou d'une organisation avec ses complémentarités.

A l'inverse, si on considère l'AIR et l'AISo, non pas comme de simples moments (successifs) dans la compréhension de l'avènement, de la stabilisation et du changement des règles dans l'histoire, mais comme des analyses qui suffisent à expliquer ou comprendre les formes institutionnelles en place au niveau territorial ou à celui d'une organisation particulière à tel moment, cette complémentarité s'efface en laissant la place à des oppositions. L'institutionnalisme holiste (AISo et AIH) se présente alors comme une approche alternative à l'AIR; puis l'AIH, comme une alternative structuraliste à l'AISo simplement ensembliste.

Cette seconde optique est celle qui remet sur le devant de la scène les affrontements. D'une part, entre les nouveaux orthodoxes qui mettent en œuvre l'AIR - comme extension ou dépassement de la PR en économie ou comme alternative à la PS en sociologie et en science politique - et les nouveaux hétérodoxes qui préconisent l'institutionnalisme holiste. D'autre part, entre les hétérodoxes modérés qui se préoccupent d'attirer sur une position intermédiaire (AISo) une majorité de chercheurs de leur discipline et les autres qui se préoccupent de construire une alternative structuraliste conséquente en $\mathrm{AIH}$. Ce n'est pas essentiellement au plan positif que l'affrontement se situe alors, mais au plan normatif. La contestation de l'AIR par les nouveaux hétérodoxes porte sur l'illusion véhiculée par cette approche lorsqu' on s'y arrête : le chercheur en science sociale serait à même de dire aux citoyens quelles sont les bonnes institutions. Les partisans de

45 Economie et Institutions $-n^{\circ} 4-1^{e}$ semestre 2004 
l'AIH, pour qui cette approche est la forme la plus complète de mise en œuvre de la PI comme alternative à la $\mathrm{PR}^{40}$, mettent de plus en exergue l'illusion que véhicule l'AISo : en raison de la cohérence systémique entre rationalité et règles, une mutation de ces dernières s'avèrerait quasiment impossible. La position qu'ils défendent est qu'on ne peut échapper à cette illusion que si la formation des règles est pensée en dynamique avec dépendance de chemin. La guillotine de Hume selon laquelle " on ne peut déduire ce qui doit être de ce qui est »- ce que confirme à sa manière l'AIH en faisant voir que l'avenir est ouvert - aurait dû régler son compte à cette seconde optique. Mais on sait pourquoi il n'en va pas ainsi. Le fait que l'affrontement occupe le devant de la scène en occultant la complémentarité tient à la réflexivité de la science sociale positive : cette connaissance n'a de sens que si elle est un facteur affectant le changement social.

\section{Références}

Amable B., Palombarini S. [2003], « Une approche néo-réaliste de l'économie politique", Communication au colloque "Institutions et conventions», La Défense, Dec.

Argyris C. et Schön D.A. [1978], Organizational learning : a theory of action perspective, Reading, Addison-Wesly.

Arrow K. J. [1973], «Information and Economic Behavior », lecture to the Fedération of Swedish Industries. Publié dans The Collected Papers, vol. 4, Basil Blackwell, 1984, p. 136-152.

Arrow K. J. [1974], The Limits of Organisations, New York, Norton. Ed. fr.: 1976, Les limites de l'organisation, Paris, PUF.

Balibar E. [1997], «Le structuralisme: méthode ou subversion des sciences sociales ", in : Andréani T. et Menahem R. (dir.), Structure, système, champ et théorie du sujet, Paris, L' Harmattan.

Batifoulier P. (sous la dir.) [2001], Théorie des conventions, Paris, Economica. Bazzoli L. [2001], L'économie politique de John R. Commons, Paris, L'Harmattan. Bessy C., Favereau O. [2003], "Institutions et économie des conventions », Cahiers d'économie politique, $\mathrm{n}^{\circ} 44$, Paris, L'Harmattan.

Billaudot B. [1996], L'ordre économique de la société moderne : un réexamen de la théorie de la régulation, Col. Théorie sociale contemporaine, Paris, L'Harmattan.

Billaudot B. [2001], Régulation et croissance. Une macroéconomie historique et institutionnelle, Paris, L'Harmattan, 304 p.

$40 \mathrm{Ou}$ comme dépassement des limites de l'AS (approche structuraliste standard).

Economie et Institutions $-n^{\circ} 4-1^{e}$ semestre 2004 
Billaudot B. [2002], «Patrimoines productifs, secteur et territoire », Géographie, Economie, Société, 4 (2002), pp. 259-303.

Billaudot B. [2004a], " Méthodologie économique », Ecole doctorale d'économie, UPMF-Grenoble.

Billaudot B. [2004b], «A propos de deux questions concernant le concept de patrimoine : de quels éléments se compose un patrimoine et quels en sont les titulaires possibles ? », Géographie, Economie, Société, volume 6 - N³ , juilletseptembre, Lavoisier, pp.291-303.

Blaug M. [1994], La méthodologie économique, $2^{\circ}$ ed., Paris, Economica.

Boltanski L. et Thevenot L. [1991], De la justification. Les économies de la grandeur, Paris, Gallimard.

Boltanski L. et Chiapello E. [1999], Le nouvel esprit du capitalisme, Paris, Gallimard.

Boudon R. [1979], La logique du social, Paris, Hachette.

Boudon R. [1985], «L'individualisme méthodologique », in : Encyclopédia Universalis, Supplément, vol. 2, Les Enjeux, pp. 644-647.

Boudon R. [1989], "Subjective rationality and the explanation of social behavior », Rationality and Society, 1, 2, pp. 171-196.

Boudon R. [2003], Raison, bonnes raisons, coll. Philosopher en sciences sociales, Paris, Presses universitaires de France.

Bourdieu E. [1998], Savoir faire, Paris, PUF.

Bourdieu P. [1980], Questions de sociologie, Paris, Les Editions de Minuit.

Bourdieu P. [1994], Raisons pratiques, Paris, Seuil.

Boyer R. [2003a], "Les analyses historiques comparatives du changement institutionnel: quels enseignements pour la théorie de la régulation? », L'Année de la régulation, $\mathrm{n}^{\circ}$ 7, 2003-2004, pp. 167-203.

Boyer R. [2003b], «Les institutions dans la théorie de la régulation », Document de travail Cepremap, $\mathrm{n}^{\circ} 2003-08$.

Boyer R. [2004], Théorie de la régulation. I. Les fondamentaux, coll. Repères, Paris, La Découverte.

Boyer R. et Saillard Y. (dir) [2002], Théorie de la régulation. L'état des savoirs, Paris, La Découverte.

Caillé A. [1986], Splendeurs et misères des sciences sociales, Genève, Droz.

Chanteau J.-P. [2003], « La dimension socio-cognitive des institutions et de la rationalité : éléments pour une approche holindividualiste », L'Année de la Régulation, vol.7, 2003-2004, pp. 49-92.

Charreaux G. [1999], «La théorie positive de l'agence : lectures et relectures ", in Koenig G. (coord. ), De nouvelles théories pour gérer l'entreprise du XXIè siècle, Paris, Economica, pp. 61-141.

Chavance B. [2001], «Organisations, institutions, système : types et niveaux de règles ", Revue d'économie industrielle, $\mathrm{n}^{\circ}$ 97-4 trim., pp. 85-102.

Cherkaoui M. [2003], « Les transitions micro-macro. Limites de la théorie du choix rationnel dans les Foundations of social theory ", Revue française de Sociologie, 44-2, avril-juin, pp. 231-254.

47 Economie et Institutions $-n^{\circ} 4-1^{\text {e }}$ semestre 2004 
Coleman J. S. [1986], «Social theory, social research and a theory of action », American journal of sociology, 91, 6, pp. 1309-1335.

Coleman J.S. [1990], Foundations of Social Theory, Cambridge (Mas.), Belknap Press of Harvard University Press.

Commons J. R. [1934], Institutional Economics. Its Place in Political Economy, The University of Wisconsin Press, 1959, 2 vol. (1 ed. 1934, Macmillan).

Defalvard H. [1992], "Critique de l'individualisme méthodologique revu par l'économie des conventions », Revue économique, $\mathrm{n}^{\circ}$ 1, janvier, pp.127-143. Defalvard H. [2000], "L'économie des conventions à l'école des institutions", Document de travail, Noisy le Grand, Centre d'étude de l'emploi, ${ }^{\circ} 2$, juillet.

Descombes V. [1979], Le même et l'autre, Paris, Editions de Minuit.

Descombes V. [1996], Les institutions du sens, Paris, Editions de Minuit.

DiMaggio P. et Powell W. [1991], The New Institutionnalism in Organizational Analysis, Chicago, The University of Chicago Press.

DiMaggio P. et Powell W. [1997], (trad. fr. de l'introduction de [1991]), « Le néo-institutionnalisme dans l'analyse des organisations », Politix. Revue des sciences sociales $d u$ politique, $\mathrm{n}^{\circ}$ 40, quatrième trimestre, pp. 113-154.

Dupuy J. -P. [1992], Introduction aux sciences sociales. Logique des phénomènes collectifs, Paris, Ellipses.

Dupuy J.-P., Eymard-Duvernay F., Favereau O., Orléan A., Salais R., Thevenot L. [1989], «L'économie des conventions », Revue économique, vol. $40 n^{\circ} 2$, mars.

Durkheim E. [1894], Les règles de la méthode sociologique, réed [1988], Paris, Flammarion.

Eymard-Duvernay F. [2002], «Pour un programme d'économie institutionnaliste », Revue économique, $\mathrm{n}^{\circ}$ 52, mars 2002, pp. 325-336.

Elias N. [1985], La société de cour, Paris, Flammarion.

Favereau O. [1995], «Conventions et régulation », in Boyer R. et Saillard Y. (dir), Théorie de la régulation. L'état des savoirs, Paris, La Découverte.

Giddens A. [1987], La constitution de la société, Paris, PUF (trad. fr. de The constitution of society, Cambridge, Polity Press, 1984).

Girard R. [1978], Des choses cachées depuis la fondation du monde, Paris, Grasset.

Goldmann L. [1977], "Structuralisme génétique en sociologie de la littérature ", in Goldmann A., Lowy M. et Sami N. (eds.), Le structuralisme génétique. L'œuvre et l'influence de Lucien Goldmann, Paris, Denoël, pp. 17-38.

Grossetti M. et Filippi M. [2004], « Proximité et relations interindividuelles », in Pecqueur B. et Zimmermann J-B. (dir), Economie de proximité, Paris, Hermes-Lavoisier.

Hall P. et Taylor R. [1996], "Political Science and the Three New Institutionnalisms », Political Studies, dec.; trad.fr.: [1997], "La science politique et les trois néo-institutionnalismes", Revue française de science politique, vol. 47, $\mathrm{n}^{\circ}$ 3-4, juin-août, pp. 469-495.

Economie et Institutions $-n^{\circ} 4-1^{e}$ semestre 2004 
Hirschman A.O. [1995], Défection et prise de parole, Paris, Fayard.

Klockhohn C. [1951], "Values and Values-orientations in the Theory of Action », in Parsons T. et Schils E., Toward a General Theory of Action, Cambridge (Mass.).

Lestienne R. [1993], Le hasard créateur, Paris, La Découverte.

Levi-Strauss C. [1991], «Introduction à l'œuvre de Marcel Mauss », in Mauss M., Sociologie et anthropologie, Paris, PUF (1 ière ed., 1950, Paris, Quadrige).

Lipietz A. [1988], « La trame, la chaîne et la régulation », Documents de travail, Cepremap, $\mathrm{n}^{\circ} 88-16$.

Lordon F. [2003], "Conatus et institutions: pour un structuralisme énergétique », L'Année de la Régulation, vol. 7, 2003-2004, pp.111-146.

Mouchot C. [1996], Méthodologie économique, Paris, Hachette.

Mäki, U., Gustafsson B. et Knudsen C. (dir.) [1993], Rationality, institutions and economic methodology, London, New York, Routledge.

North D. [1990], Institutions, Institutional Change and Economic Performance, Cambridge, Cambridge University Press.

Orlean A. [1994], « Vers un modèle général de la coordination économique par les conventions ", in Orlean A. (dir.), Analyse économique des conventions, Paris, PUF, pp. 9-40.

Peirce C. S. [1879], «Comment rendre nos idées claires », in Textes cartésiens, Paris, Aubier, 1984, pp. 287-308.

Piaget J. [1968], Le structuralisme, Paris, PUF.

Pirou G. [1939], Les nouveaux courants de la théorie économique aux Etats-Unis. Tome II : L'économie institutionnelle, ( $2^{\circ} \mathrm{ed}$.), Paris, Domat-Montchrestien.

Postel N. [2003], Les règles dans la pensée économique contemporaine, Paris, CNRS Editions, $260 \mathrm{p}$.

Salais R. [1998], "A la recherche du fondement conventionnel des institutions», in Salais R. (eds.), Institutions et conventions, Paris, Editions de l'EHESS, pp.255-291.

Sapir J. [2003], Les trous noirs de la science économique, Paris, Editions poche. Servais O. [2000], «Les modèles d'action dans la théorie de la régulation. Habitus, rationalité, routine », Economies et Sociétés, Tome XXXIV, n 1, Janv. 2000, Série Théorie de la régulation, $R, n^{\circ} 11$.

Servais O. [2002], "Croyances et dispositions dans la théorie de la régulation", Journées d'étude Institutionnalismes et évolutionnismes. Confrontations autour de perspectives empiriques, Lyon, 2 et 3 dec. 2002.

Scitovsky T. [1978], L'économie sans joie, Paris, Calman Levy.

Simmel G. [1987], Philosophie de l'argent, Paris, PUF [Trad. fr. de Philosophie des Geldes, 1977, Dunker \& Humblot, Berlin].

Simon H. [1976], «From Substantive to Procedural Rationality », in Latsis S. (dir.), Method and Appraisal in Economics, Cambridge, Cambridge University Press, pp.129-148.

Srinivasan R. [1984], Entretiens avec Svami Prajnanpad, Paris, L'Originel.

49 Economie et Institutions $-\mathrm{n}^{\circ} 4-1^{\mathrm{e}}$ semestre 2004 
Steiner P. [2003], « Les Foundations de James S. Coleman : une introduction », Revue française de Sociologie, 44-2, 2003, pp. 205-229.

Théret B. [2000a], «Institutions et institutionnalismes : vers une convergence des conceptions de l'institution ? ", in Tallard M., Théret B. et Uri D. (dir.), Innovations institutionnelles et territoires, Paris, L'Harmattan.

Théret B. [2000b], «Nouvelle économie institutionnelle, Economie des conventions et Théorie de la régulation : vers une synthèse institutionnaliste ?», La lettre de la régulation, dec., pp. 1-4.

Théret B. [2003], "Institutionnalismes et structuralismes: oppositions, substitutions ou affinités électives?", Cahiers d'économie politique, $\mathrm{n}^{\circ} 44$, L'Harmattan.

Ughetto P. [2003], « Action et interprétation: les bases d'un rapprochement paradigmatique au sein des analyses hétérodoxes », Forum de la Régulation 2003, oct. , Paris.

Veblen T. [1971], Les ingénieurs et le capitalisme, Paris, Gordon et Breach Publications Gramma.

Weber M. [1995], Economie et société, Paris, Agora pocket, Plon.

Williamson O. [1996], "Efficiency, power, authority and economic organisation ", Groenevegen J. (dir.), Transaction cost, economics and beyong, Boston, Kluwer Academic Publishers. 